\title{
Improved signal detection for two-way wireless relaying networks under imperfect time synchronisation
}

\author{
Mohammed Taha O El Astal ${ }^{*}$ and Jan Corné Olivier
}

\begin{abstract}
Most research on two-way wireless relaying networks (TWRNs) assumes perfect time synchronisation between the terminals and among the relays. This is, however, difficult to accomplish as the terminals and relays are randomly located. The lack of synchronisation causes inter-symbol interference which introduces a serious impairment and negatively impacts the network's performance. In this article, an enhanced interference cancellation (EIC)-based detector is proposed to mitigate the experienced performance degradation while maintaining low complexity. It uses an innovative iterative approach and also benefits of interference components of currently detected symbols using a modified maximum likelihood (ML) scheme. As a result, it shows significant performance improvement compared to the conventional detector under different conditions of imperfect synchronisation. In addition, two existing interference cancellation detectors that are originally proposed for one-way WRNs are extended to TWRNs, even though the timing error among the terminals is a unique issue for TWRNs and never occurs in one-way networks. Unlike the existing literature, the proposed detectors apply to networks with an arbitrary number of multiple-antenna relays. Numerical simulation is provided in terms of bit error rate, to show the improvement in performance when compared to the conventional detector. Moreover, it is shown that the EIC detector outperforms the extended detectors and provides 3 to $4 \mathrm{~dB}$ gain under moderate condition of imperfect synchronisation.
\end{abstract}

Keywords: Two-way wireless relaying networks; Imperfect synchronisation; Parallel interference cancellation; Successive interference cancellation

\section{Introduction}

Recently, various data and voice services have been incorporated over modern wireless communication networks, such as the Internet, video calling and gaming. These networks are required to provide both high speed and quality services to gain consumer satisfaction. The use of multiple-antenna nodes in communications is a known technology to increase capacity and improve reliability, especially when compared with single-antenna node networks. This is known as multiple-input multiple-output (MIMO) technology. This is, however, infeasible in some applications due to the size, cost and environmental constraints [1]. The potential spatial diversity that arises from the presence of multiple distributed nodes in a network

*Correspondence: mohammed.elastal@utas.edu.au

School of Engineering and ICT, University of Tasmania, Private Bag 65, Hobart, Tasmania, Australia can be exploited if suitable cooperation is arranged among these nodes [2]. This creates a virtual MIMO network. In [3], the use of a distributed space-time block code (D-STBC) is suggested to exploit the spatial diversity effectively and hence improve the link reliability significantly. In addition, the D-STBCs increase the spectrum efficiency of the network due to the allowance of simultaneous transmission from relays. However, this network is still limited by half-duplex constraints. In other words, to exchange a data packet between the connection terminals, four time slots are required. In contrast, two-way wireless relaying networks (TWRNs) have been proposed to halve this number of required time slots. This is experienced as the terminals are allowed to transmit simultaneously too [4]. Due to the promising bandwidth efficiency, the TWRN has drawn much interest in the literature [5-9]. However, in most pioneering research, a perfect timing synchronisation between the terminals' signal in the first

\section{Springer}

(c) 2014 El Astal and Olivier; licensee Springer. This is an Open Access article distributed under the terms of the Creative Commons Attribution License (http://creativecommons.org/licenses/by/4.0), which permits unrestricted use, distribution, and reproduction in any medium, provided the original work is properly credited. 
phase of transmission as well as among the relays in the next phase of transmission is assumed. Unfortunately, this assumption is unrealistic or at the very least it imposes conditions that will be difficult or even impossible to achieve in a real-world network [10,11]. Consequently, this makes the channel appears dispersive, and hence, the structure of the code matrix is compromised $[11,12]$. This introduces a serious impairment and negatively impacts the network performance due to the rising inter-symbol interference (ISI).

\subsection{Prior work}

Unlike one-way WRNs, two forms of imperfect timing synchronisation exist in TWRNs. This is due to the dispersed nature of both the terminals and relays. So, this different timing error is introduced between the received signals of terminals at the relays and among the received signals of relays at the terminals too. In [13], the authors consider the timing error among the terminals and conclude that a significant performance degradation is introduced but no mitigation strategy is offered. In [14], a preamble structure is proposed for efficient synchronisation among the terminals only. A delay-tolerant distributed space-time trellis code is proposed to conserve high performance even under imperfect synchronisation in [15]. However, this code functions in the binary field, meaning it cannot be employed in amplify-and-forward (AF) TWRNs. In [16-18], robust schemes against the relaying timing error are proposed. They use a sufficient cyclic prefix and/or performing physical-layer network coding. These schemes may involve much higher complexity and introduce a significant control overhead. In addition, the inter-block interference among the relays is not considered. In one-way WRNs, a number of lowcomplexity methods have been proposed, notably parallel interference cancellation (PIC) and successive interference cancellation (SIC) $[11,12]$. Although the timing error among the terminals is a unique issue for TWRNs and never occurs in one-way networks, these methods can be extended to TWRNs.

\subsection{Our contribution}

In summary and with respect to the current literature, our contributions are threefold:

- A mathematical model is derived under a condition of imperfect synchronisation for TWRNs with $N$ $R_{a}$-antenna relays, where $N, R_{a} \in \mathbb{N}^{+}$. Also, it can be used to evaluate a model for either orthogonal or quasi-orthogonal linear dispersion STBCs.

- An enhanced interference cancellation (EIC)-based detector is proposed for asynchronous TWRNs to mitigate the experienced performance degradation. It conducts the interference cancellation process in an innovative iterative approach. It orders the cancellation process according to the estimated reliability of the available detected symbols. Unlike $[11,12]$, this detector shows higher performance, particularly in the first iteration, as it reduces the reliance on low-performance symbols. Moreover, the EIC benefits from interference components of currently detected symbols using a modified maximum likelihood (ML) scheme.

- Both PIC- and SIC-based one-way detectors are extended to work in TWRNs. They show a significant performance improvement compared to the conventional detectors.

\subsection{Notations}

In the rest of the paper, non-bold letters, bold lowercase letters and bold capital letters will designate scalars, vectors and matrices, respectively. For matrices $\mathbf{A}, \mathbf{A}^{H}$, $\mathbf{A}^{*}$ and $\mathbf{A}^{T}$ denote the hermitian, the conjugate and the transpose of $\mathbf{A}$, respectively. $\Psi_{1}(\mathbf{A}, a, b)$ denotes the result of shifting down each $b$ row of $\mathbf{A}$ by $a$ rows individually with zero padding. Similarly, $\Psi_{2}(\mathbf{A}, a, b)$ denotes the result of shifting $\mathbf{A}$ as with $\Psi_{1}(\mathbf{A}, a, b)$ but in an upward direction. For a vector $\mathbf{v}, \overline{\mathbf{v}}=\left[\Re(\mathbf{v})^{T}, \Im(\mathbf{v})^{T}\right]^{T}$, where $\Re($. and $\Im($.$) indicate the real and imaginary part, respectively.$ The operator $\otimes$ is the Kronecker product. A Gaussian distribution is denoted by $\mathcal{C N}\left(\mu, \Gamma^{2}\right)$, with corresponding mean $\mu$ and diagonal covariance matrix $\Gamma^{2}$ with value $\sigma^{2}$.

\section{Network model under a condition of imperfect synchronisation}

A TWRN consist of two terminals $\mathbb{T}_{1}$ and $\mathbb{T}_{2}$ that exchange data through $N$ relay nodes: $R_{n} n=1 \cdots N$ is considered, see Figure 1. The terminals are single-antenna nodes while the relays are equipped each with $R_{a}, R_{a} \in$ $\mathbb{N}^{+}$. The relays use linear dispersion STBC and employ an AF relaying protocol. The transmission through the network is conducted in two phases: in the first phase,

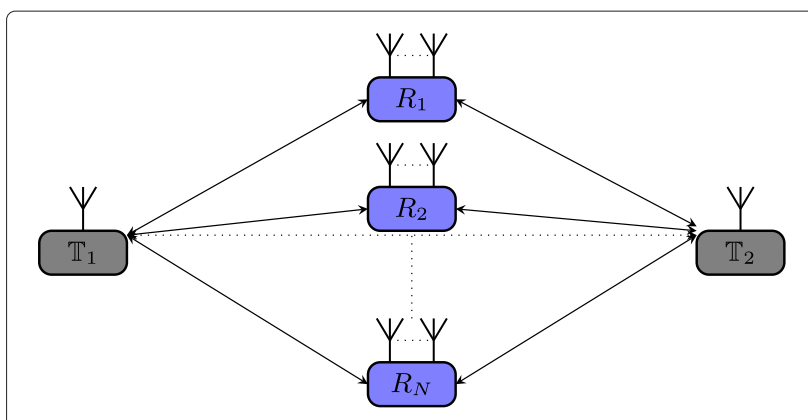

Figure 1 Two-way wireless relaying network (TWRN) model. 
the information is broadcast from the terminals to the relays simultaneously. In the second phase, each relay amplifies an encoded version of its received symbols and then forwards it to the terminals. The total transmission power for the entire network is denoted by $P$ and is evenly divided between both phases and nodes. The channel through the network is assumed to be a quasi-static Rayleigh fading channel. Like most TWRNs, it is assumed that the channel coefficients remain constant for $2 J$ consecutive channel uses, where $J$ is the number of symbols exchanged each transmission through the network [4-6]. The relays are assumed to be completely blind while perfect channel-state information (CSI) is assumed only at the terminals.

Due to the factors such as different timing and different propagation delays, the accurate synchronisation that is assumed among relays and between the terminals is difficult to achieve [10]. Here, the TWRN is generally modelled under a condition of imperfect synchronisation. Then, three instances of the general modelled are evaluated for the following networks: (1) two dualantenna relays using the novel quasi-orthogonal STBC (QO-STBC) of [5]; (2) two single-antenna relays using the orthogonal Alamouit's STBC of [19]; and (3) four singleantenna relays using the QO-STBC of [20]. These models are used later to examine the effectiveness of the proposed detectors.

\subsection{General network model}

As mentioned, the transmission through TWRNs is conducted in two phases.

Broadcasting phase (phase 1): the terminals transmit simultaneously their $M$-ary PSK-/QAM-modulated symbols that are denoted by $\mathbf{x}(i)=[x(i, 1), . ., x(i, j), . ., x(i, J)]^{T}$ and $\mathbf{y}(i)=[y(i, 1), . ., y(i, j), . ., y(i, J)]^{T}$, where $i$ denotes the information block index and $J$ is the number of symbols of each terminal in the broadcasting phase. Due to the different timing and propagation delays, the transmitting symbols arrive to the relays with a different delay: $\tau_{n}$. Similar to $[11,12]$, a condition of $\tau_{n} \in[0,0.5 T]$ is assumed, where $T$ is the symbol signalling period. Thus, the received vector at $R_{n}$ can be expressed by

$$
\begin{aligned}
\underset{\left(R_{a} \times J\right) \times 1}{\mathbf{r}_{n}(i)=} & \sqrt{P_{2}} \underset{\left(R_{a} \times J\right) \times J J \times 1}{\mathbf{G}_{n}(0)} \mathbf{x}(i)+\sqrt{P_{2}} \underset{\left(R_{a} \times J\right) \times J J \times 1}{\mathbf{H}_{n}(0)} \mathbf{\mathbf { y }}(i)+\underset{\left(R_{a} \times J\right) \times 1}{\mathbf{q}_{n}(i)} \\
& +\underset{\left(R_{a} \times J\right) \times 1}{\mathbf{v}_{n}(i)},
\end{aligned}
$$

with

$$
\begin{aligned}
\mathbf{q}_{n}(i)= & \sqrt{P_{1}}\left(\mathbf{g}_{n}(1) \otimes(\mathbf{Q x}(i)+\mathbf{N x}(i-1))\right) \\
& +\sqrt{P_{2}}\left(\mathbf{h}_{n}(1) \otimes(\mathbf{Q y}(i)+\mathbf{N y}(i-1))\right),
\end{aligned}
$$

where $P_{1}$ and $P_{2}$ are the power dedicated for the broadcasting phase for $\mathbb{T}_{1}$ and $\mathbb{T}_{2}$, respectively.

$$
\begin{aligned}
& \underset{J \times J}{\mathbf{Q}}=\left[\begin{array}{cc}
\mathbf{0}_{1 \times(J-1)} & 0 \\
\mathbf{I}_{J-1} & \mathbf{0}_{(J-1) \times 1}
\end{array}\right] \text { and } \\
& \underset{J \times J}{\mathbf{N}}=\left[\begin{array}{cc}
\mathbf{0}_{1 \times(J-1)} & 1 \\
\mathbf{0}_{(J-1) \times(J-1)} & \mathbf{0}_{(J-1) \times 1}
\end{array}\right] .
\end{aligned}
$$

$\mathbf{G}_{n}(0)=\mathbf{I}_{J} \otimes \mathbf{g}_{n}(0), \mathbf{H}_{n}(0)=\mathbf{I}_{J} \otimes \mathbf{h}_{n}(0)$. Both $\mathbf{g}_{n}(0)=\left[g_{n, 1}(0), \ldots\right.$, $\left.g_{n, R_{a}(0)}\right]^{T}$ and $\mathbf{h}_{n}(0)=\left[h_{n, 1}(0), \ldots, h_{n, R_{a}}(0)\right]^{T}$ are the current time slot channel coefficient vectors for the $\mathbb{T}_{1}$-and$R_{n}$ and $\mathbb{T}_{2}$-and- $R_{n}$ links, respectively; $\mathbf{g}_{n}(v)$ and $\mathbf{h}_{n}(v)$ for $v=1, \ldots, \infty$ represent the composite effects of time delay and the pulse shaping waveform (PSW) for the mentioned links. As they are much less dominant, both $\mathbf{g}_{n}(v)$ and $\mathbf{g}_{n}(v)$ for $v=2, \ldots, \infty$ can be truncated [12]. The strength of $\mathbf{g}_{n}(1)$ or $\mathbf{h}_{n}$ (1) can be represented by (3). It is worth noting that regardless of which PSW is used, $\alpha_{n}=0$ for $\tau_{n}=0$, and $\alpha_{n}=1(0 \mathrm{~dB})$ for $\tau_{n}=0.5 T$, due to the symmetry of the PSW.

$$
\alpha_{n}=\frac{\left\|\mathbf{g}_{n}(1)\right\|^{2}}{\left\|\mathbf{g}_{n}(0)\right\|^{2}} .
$$

Assuming an availability of full-duplex terminals, a direct transmission (DT) link between the terminals exists. This case is not common in TWRNs, and hence, it is considered as an optional case. The DT links are modelled by

$$
\mathbf{r}_{\mathrm{DT}, 1}(i)=\sqrt{P_{2}} f \underset{J \times 1}{\mathbf{y}}(i)+\underset{J \times 1}{\boldsymbol{\delta}_{1}}(i),
$$

and

$$
\underset{J \times 1}{\mathbf{r}_{\mathrm{DT}, 2}}(i)=\sqrt{P_{1}} f \underset{J \times 1}{\mathbf{x}}(i)+\underset{J \times 1}{\boldsymbol{\delta}_{2}}(i),
$$

where $f(f \sim \mathcal{C N}(0,1))$ is the channel coefficient for the link between $\mathbb{T}_{1}$ and $\mathbb{T}_{2}$. The vector $\delta_{k}, \delta_{k} \sim \mathcal{C N}(0,1)$, is the noise vector at $\mathbb{T}_{k}$. The detection result of the DT links, denoted later by $\hat{\mathbf{x}}_{\mathrm{DT}}(i)$ and $\hat{\mathbf{y}}_{\mathrm{DT}}(i)$, can be carried through a conventional ML detector $[11,20]$.

Relaying phase (phase 2): according to the used code, each relay combines the received symbol vector into a new transmitting symbols $R_{a} \times J$ vector $\mathbf{t}_{n}(i)$ using

$$
\begin{aligned}
\underset{\mathbf{t}_{n}(i, j)}{\mathbf{R}_{a} \times 1} & =\beta\left(\underset{R_{a} \times\left(R_{a} \times J\right)\left(R_{a} \times J\right) \times 1}{\mathbf{A}_{n}^{(j)}} \underset{R_{a} \times\left(R_{a} \times J\right)}{\mathbf{r}_{n}(i)}+\underset{\left(R_{a} \times J\right) \times 1}{\mathbf{B}_{n}^{(j)}}\right. \\
\forall j & =1, \ldots, J,
\end{aligned}
$$

where $\mathbf{A}_{n}^{(j)}$ and $\mathbf{B}_{n}^{(j)}$ are the encoding matrices that are used by $R_{n}$ to construct its corresponding part of the employed code in a distributive manner. $\beta$ is the power amplification gain that is used to normalise the average power per antenna of each relay to be $p_{r}$. These resulting symbols are then forwarded simultaneously from all participating 
relays to the terminals. Again, interference terms due to the imperfect time synchronisation among relays arise at the terminals (see Figure 2).

Due to the similarity, only the received signal at $\mathbb{T}_{1}$ is considered, which is expressed as

$$
\begin{aligned}
\underset{J \times 1}{\mathbf{z}_{1}(i)=} & \sqrt{P_{1}} \beta(\overbrace{\mathbf{A}_{g(0)} \mathbf{G}(0)}^{\mathbf{C}_{1}} \mathbf{x}(i)+\overbrace{\mathbf{B}_{g(0)} \mathbf{G}^{*}(0)}^{\mathbf{D}_{1}} \mathbf{x}^{*}(i)) \\
& +\sqrt{P_{2}} \beta(\overbrace{\mathbf{A}_{g(0)} \mathbf{H}(0)}^{\mathbf{C}_{2}} \mathbf{y}(i)+\overbrace{\mathbf{B}_{g(0)} \mathbf{H}^{*}(0)}^{\mathbf{D}_{2}} \mathbf{y}^{*}(i)) \\
& +\mathbf{q}_{\mathbb{T}_{1}}(i)+\zeta_{1}(i),
\end{aligned}
$$

where

$$
\begin{aligned}
\underset{J \times\left(J \times N \times R_{a}\right)}{\mathbf{A}_{g(0)}}= & {\left[\begin{array}{cccc}
\mathbf{g}_{1}^{T}(0) \mathbf{A}_{1}^{(1)} & \mathbf{g}_{2}^{T}(0) \mathbf{A}_{2}^{(1)} & \cdots & \mathbf{g}_{N}^{T}(0) \mathbf{A}_{N}^{(1)} \\
\mathbf{g}_{1}^{T}(0) \mathbf{A}_{1}^{(2)} & \mathbf{g}_{2}^{T}(0) \mathbf{A}_{2}^{(2)} & \cdots & \mathbf{g}_{N}^{T}(0) \mathbf{A}_{N}^{(2)} \\
\vdots & \ddots & \ddots & \vdots \\
\mathbf{g}_{1}^{T}(0) \mathbf{A}_{1}^{(J)} & \mathbf{g}_{2}^{T}(0) \mathbf{A}_{2}^{(J)} & \cdots & \mathbf{g}_{N}^{T}(0) \mathbf{A}_{N}^{(J)}
\end{array}\right], } \\
\underset{J \times\left(J \times N \times R_{a}\right)}{\mathbf{B}_{g(0)}}= & {\left[\begin{array}{cccc}
\mathbf{g}_{1}^{T}(0) \mathbf{B}_{1}^{(1)} & \mathbf{g}_{2}^{T}(0) \mathbf{B}_{2}^{(1)} & \cdots & \mathbf{g}_{N}^{T}(0) \mathbf{B}_{N}^{(1)} \\
\mathbf{g}_{1}^{T}(0) \mathbf{B}_{1}^{(2)} & \mathbf{g}_{2}^{T}(0) \mathbf{B}_{2}^{(2)} & \cdots & \mathbf{g}_{N}^{T}(0) \mathbf{B}_{N}^{(2)} \\
\vdots & \ddots & \ddots & \vdots \\
\mathbf{g}_{1}^{T}(0) \mathbf{B}_{1}^{(J)} & \mathbf{g}_{2}^{T}{ }_{(0)} \mathbf{B}_{2}^{(J)} & \cdots & \mathbf{g}_{N}^{T}(0) \mathbf{B}_{N}^{(J)}
\end{array}\right], }
\end{aligned}
$$

$\mathbf{G}(0)=\left[\mathbf{G}_{1}^{T}(0), \cdots, \mathbf{G}_{N}^{T}(0)\right]^{T}$, and $\mathbf{H}(0)=\left[\mathbf{H}_{1}^{T}(0), \cdots, \mathbf{H}_{N}^{T}(0)\right]^{T}$. The noise terms at $\mathbb{T}_{1}$ can be modelled equivalently as

$$
\begin{aligned}
\underset{J \times 1}{\zeta_{1}=} & \beta\left(\left(\mathbf{A}_{g(0)}+\Psi_{1}\left(\widehat{\mathbf{A}}_{g(1)}, 1, J\right)\right) \mathbf{v}\right. \\
& \left.+\left(\mathbf{B}_{g(0)}+\Psi_{1}\left(\widehat{\mathbf{B}}_{g(1)}, 1, J\right)\right) \mathbf{v}^{*}\right)+\eta_{1},
\end{aligned}
$$

where $\mathbf{v}=\left[\mathbf{v}_{1}^{T}, \cdots, \mathbf{v}_{N}^{T}\right]^{T}$ and $\eta_{1} \in \mathcal{C N}(0,1)$. Both $\mathbf{A}_{g(1)}$ and $\mathbf{B}_{g(1)}$ are formed using $\mathbf{g}_{n}(1)$ instead of $\mathbf{g}_{n}(0)$ as follows:

$$
\begin{aligned}
\underset{J \times\left(J \times N \times R_{a}\right)}{\mathbf{A}_{g(1)}}= & {\left[\begin{array}{cccc}
\mathbf{g}_{1}^{T}(1) \mathbf{A}_{1}^{(1)} & \mathbf{g}_{2}^{T}(1) \mathbf{A}_{2}^{(1)} & \cdots & \mathbf{g}_{N}^{T}(1) \mathbf{A}_{N}^{(1)} \\
\mathbf{g}_{1}^{T}{ }_{(1)} \mathbf{A}_{1}^{(2)} & \mathbf{g}_{2}^{T}(1) \mathbf{A}_{2}^{(2)} & \cdots & \mathbf{g}_{N}^{T}(1) \mathbf{A}_{N}^{(2)} \\
\vdots & \ddots & \ddots & \vdots \\
\mathbf{g}_{1}^{T}(1) \mathbf{A}_{1}^{(J)} & \mathbf{g}_{2}^{T}(1) \mathbf{A}_{2}^{(J)} & \cdots & \mathbf{g}_{N}^{T}(1) \mathbf{A}_{N}^{(J)}
\end{array}\right], } \\
\underset{J \times\left(J \times N \times R_{a}\right)}{\mathbf{B}_{g(1)}}= & {\left[\begin{array}{ccccc}
\mathbf{g}_{1}^{T}{ }_{(1)} \mathbf{B}_{1}^{(1)} & \mathbf{g}_{2}^{T}(1) \mathbf{B}_{2}^{(1)} & \cdots & \mathbf{g}_{N}^{T}(1) \mathbf{B}_{N}^{(1)} \\
\mathbf{g}_{1}^{T}{ }_{(1)} \mathbf{B}_{1}^{(2)} & \mathbf{g}_{2}^{T}(1) \mathbf{B}_{2}^{(2)} & \cdots & \mathbf{g}_{N}^{T}(1) \mathbf{B}_{N}^{(2)} \\
\vdots & \ddots & \ddots & \vdots \\
\mathbf{g}_{1}^{T}(1) \mathbf{B}_{1}^{(J)} & \mathbf{g}_{2}^{T}(1) \mathbf{B}_{2}^{(J)} & \cdots & \mathbf{g}_{N}^{T}(1) \mathbf{B}_{N}^{(J)}
\end{array}\right] . }
\end{aligned}
$$

All interference terms received at $\mathbb{T}_{1}$ that resulted due to the imperfect synchronisation in the two phases of transmission can be model as

$$
\mathbf{q}_{\mathbb{T}_{1}}(i)=\mathbf{q}_{0, x}(i)+\mathbf{q}_{1, x}(i)+\mathbf{q}_{0, y}(i)+\mathbf{q}_{1, y}(i),
$$

with

$$
\begin{aligned}
\mathbf{q}_{0, x}(i)= & \sqrt{P_{1}} \beta\left(\left(\mathbf{A}_{g(0)} \Psi_{1}\left(\mathbf{G}(1), R_{a}, R_{a} J\right)+\Psi_{1}\left(\mathbf{A}_{g(1)}, 1, J\right)\right.\right. \\
& \left.\times\left(\mathbf{G}(0)+\Psi_{1}\left(\mathbf{G}(1), R_{a}, R_{a} J\right)\right)\right) \mathbf{x}(i) \\
& +\left(\mathbf{B}_{g(0)} \Psi_{1}\left(\mathbf{G}^{*}(1), R_{a}, R_{a} J\right)+\Psi_{1}\left(\mathbf{B}_{g(1)}, 1, J\right)\right. \\
& \left.\left.\times\left(\mathbf{G}^{*}(0)+\Psi_{1}\left(\mathbf{G}^{*}(1), R_{a}, R_{a} J\right)\right)\right) \mathbf{x}^{*}(i)\right)
\end{aligned}
$$

$$
\begin{aligned}
\mathbf{q}_{1, x}(i)= & \sqrt{P_{1}} \beta\left(\left(\mathbf{A}_{g(0)} \Psi_{2}\left(\mathbf{G}(1), R_{a}(J-1), R_{a} J\right)\right.\right. \\
& \left.+\Psi_{2}\left(\mathbf{A}_{g(1)}, J-1, J\right) \mathbf{G}(0)\right) \mathbf{x}(i-1) \\
& +\left(\mathbf{B}_{g(0)} \Psi_{2}\left(\mathbf{G}(1)^{*}, R_{a}(J-1), R_{a} J\right)\right. \\
& \left.+\Psi_{2}\left(\mathbf{B}_{g(1)}, J-1, J\right) \mathbf{G}^{*}(0)\right) \mathbf{x}^{*}(i-1) \\
& +\Psi_{2}\left(\mathbf{A}_{g(1)}, J-1, J\right) \Psi_{2}\left(\mathbf{G}(1), R_{a}(J-1), R_{a} J\right) \mathbf{x}(i-2) \\
& \left.+\Psi_{2}\left(\mathbf{B}_{g(1)}, J-1, J\right) \Psi_{2}\left(\mathbf{G}(1)^{*}, R_{a}(J-1), R_{a} J\right) \mathbf{x}^{*}(i-2)\right),
\end{aligned}
$$

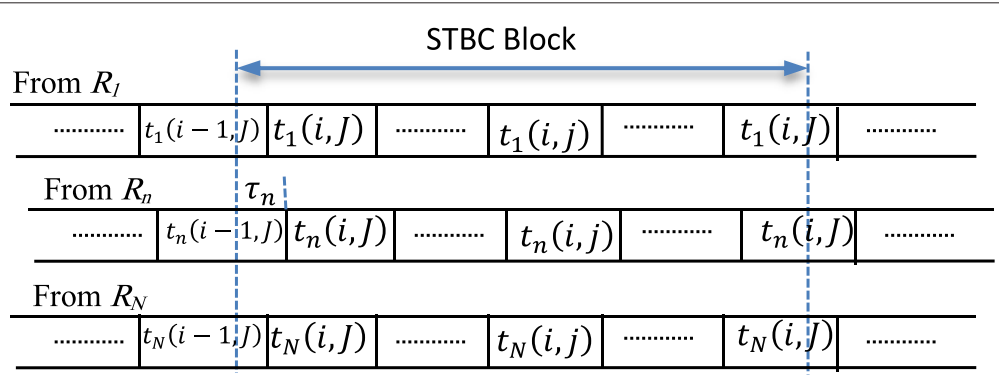

Figure 2 The relays' signal at $\mathbb{T}_{1}$ under imperfect synchronisation. 


$$
\left.\begin{array}{c}
=\sqrt{P_{2}} \beta(\overbrace{\left(\mathbf{A}_{g(0)} \Psi_{1}\left(\mathbf{H}(1), R_{a}, R_{a} J\right)+\Psi_{1}\left(\mathbf{A}_{g(1)}, 1, J\right)\left(\mathbf{H}(0)+\Psi_{1}\left(\mathbf{H}_{(1)}, R_{a}, R_{a} J\right)\right)\right)}^{\mathbf{q}_{0, y}(i)} \mathbf{y}(i) \\
+\overbrace{\left(\mathbf{B}_{g(0)} \Psi_{1}\left(\mathbf{H}^{*}(1), R_{a}, R_{a} J\right)+\Psi_{1}\left(\mathbf{B}_{g(1)}, 1, J\right)\left(\mathbf{H}^{*}(0)+\Psi_{1}\left(\mathbf{H}^{*}(1), R_{a}, R_{a} J\right)\right)\right)}^{D_{3}} \mathbf{y}^{*}(i)
\end{array}\right),
$$

and

$$
\begin{aligned}
\mathbf{q}_{1, y}(i)= & \sqrt{P_{2}} \beta\left(\left(\mathbf{A}_{g(0)} \Psi_{2}\left(\mathbf{H}(1), R_{a}(J-1), R_{a} J\right)\right.\right. \\
& \left.+\Psi_{2}\left(\mathbf{A}_{g(1)}, J-1, J\right) \mathbf{H}(0)\right) \mathbf{y}(i-1) \\
& +\left(\mathbf{B}_{g(0)} \Psi_{2}\left(\mathbf{H}(1)^{*}, R_{a}(J-1), R_{a} J\right)\right. \\
& \left.+\Psi_{2}\left(\mathbf{B}_{g(1)}, J-1, J\right) \mathbf{H}^{*}(0)\right) \mathbf{y}^{*}(i-1) \\
& +\Psi_{2}\left(\mathbf{A}_{g(1)}, J-1, J\right) \Psi_{2}\left(\mathbf{H}(1), R_{a}(J-1), R_{a} J\right) \mathbf{y}(i-2) \\
& \left.+\Psi_{2}\left(\mathbf{B}_{g(1)}, J-1, J\right) \Psi_{2}\left(\mathbf{H}(1)^{*}, R_{a}(J-1), R_{a} J\right) \mathbf{y}^{*}(i-2)\right),
\end{aligned}
$$

where $\mathbf{G}(1)=\left[\mathbf{G}_{1}^{T}(1), \cdots, \mathbf{G}_{N}^{T}(1)\right]^{T}, \mathbf{H}(1)=\left[\mathbf{H}_{1}^{T}(1), \cdots, \mathbf{H}_{N}^{T}(1)\right]^{T}$, $\mathbf{G}_{n}(1)=\mathbf{I}_{J} \otimes \mathbf{g}_{n}(1)$, and $\mathbf{H}_{n}(1)=\mathbf{I}_{J} \otimes \mathbf{h}_{n}(1)$. With some algebraic manipulations, Equation 7 can be rearranged and rewritten for detection as

$$
\begin{aligned}
& \underset{2 J \times 1}{\overline{\mathbf{z}}_{1}(i)}=\sqrt{P_{1}} \beta \mathbf{G}_{\mathbf{G}} \overline{\mathbf{x}}(i)+\sqrt{P_{2}} \beta \mathbf{G}_{\mathbf{H} \times 1} \overline{\mathbf{y}}(i)+\mathbf{G}_{\mathbf{q} \times 1} \overline{\mathbf{y}}(i)+\underset{2 J \times 1}{\overline{\mathbf{q}}_{1, y}(i)} \\
& +\underset{2 J \times 1}{\overline{\mathbf{q}}_{0, x}(i)}+\underset{2 J \times 1}{\overline{\mathbf{q}}_{1, x}(i)}+\underset{2 J \times 1}{\bar{\zeta}_{1}(i),}
\end{aligned}
$$

where

$$
\begin{gathered}
\underset{2 J \times 2 J}{\mathbf{G}_{\mathbf{G}}}=\left[\begin{array}{cc}
\Re\left(\mathbf{C}_{1}+\mathbf{D}_{1}\right) & \Im\left(-\mathbf{C}_{1}+\mathbf{D}_{1}\right) \\
\Im\left(\mathbf{C}_{1}+\mathbf{D}_{1}\right) & \Re\left(\mathbf{C}_{1}-\mathbf{D}_{1}\right)
\end{array}\right], \\
\underset{2 J \times 2 J}{\mathbf{G}_{\mathbf{H}}}=\left[\begin{array}{cc}
\Re\left(\mathbf{C}_{2}+\mathbf{D}_{2}\right) & \Im\left(-\mathbf{C}_{2}+\mathbf{D}_{2}\right) \\
\Im\left(\mathbf{C}_{2}+\mathbf{D}_{2}\right) & \Re\left(\mathbf{C}_{2}-\mathbf{D}_{2}\right)
\end{array}\right],
\end{gathered}
$$
and

$$
\underset{2 J \times 2 J}{\mathbf{G}_{\mathbf{q}}}=\left[\begin{array}{cc}
\Re\left(\mathbf{C}_{3}+\mathbf{D}_{3}\right) & \Im\left(-\mathbf{C}_{3}+\mathbf{D}_{3}\right) \\
\Im\left(\mathbf{C}_{3}+\mathbf{D}_{3}\right) & \Re\left(\mathbf{C}_{3}-\mathbf{D}_{3}\right)
\end{array}\right] .
$$

It is worth noting that the interference term $\mathbf{q}_{0, y}(i)$ is written in terms of transmitting symbols in (17) as the proposed detector requires it to be in this form.

\subsection{Two dual-antenna relay TWRNs using QO-STBC}

In [5], a novel class of D-QO-STBC is proposed for TWRNs with a number $N$ of dual-antenna relays. This code class is promising as it can achieve the maximum coding gain and the optimal diversity gain while maintaining low decoding complexity. For TWRNs employing the mentioned codes, the general network model is evaluated under imperfect synchronisation, meaning that $R_{a}=2$ and $N=2$. The encoding matrices used by this network are shown above the Equation 18 of [5]. The data exchanged is $\mathbf{x}(i)=[\mathbf{x}(i, 1), \ldots, \mathbf{x}(i, 4)]^{T}$ and $\mathbf{y}(i)=[\mathbf{y}(i, 1), \ldots, \mathbf{y}(i, 4)]^{T}$. The instance model can be represented as

$$
\begin{aligned}
\mathbf{C}_{1}= & {\left[\begin{array}{cccc}
\mathbf{g}_{1}^{T}(0) \mathbf{g}_{1}(0) & 0 & \mathbf{g}_{2}^{T}(0) \mathbf{g}_{2}(0) & 0 \\
0 & \mathbf{g}_{1}^{T}(0) \mathbf{g}_{1}(0) & 0 & \mathbf{g}_{2}^{T}(0) \mathbf{g}_{2}(0) \\
\mathbf{g}_{2}^{T}(0) \mathbf{g}_{2}(0) & 0 & \mathbf{g}_{1}^{T}(0) \mathbf{g}_{1}(0) & 0 \\
0 & \mathbf{g}_{2}^{T}(0) \mathbf{g}_{2}(0) & 0 & \mathbf{g}_{1}^{T}(0) \mathbf{g}_{1}(0)
\end{array}\right], } \\
\mathbf{D}_{1}= & {\left[\begin{array}{cccc}
0 & \mathbf{g}_{1}^{T}(0) \mathbf{J} \mathbf{g}_{1}^{*}(0) & 0 & \mathbf{g}_{2}^{T}(0) \mathbf{J} \mathbf{g}_{2}^{*}(0) \\
\mathbf{g}_{1}^{H}(0) \mathbf{J} \mathbf{g}_{1(0)} & 0 & \mathbf{g}_{2}^{H}(0) \mathbf{J} \mathbf{g}_{2}(0) & 0 \\
0 & \mathbf{g}_{2}^{T}(0) \mathbf{J} \mathbf{g}_{2}^{*}(0) & 0 & \mathbf{g}_{1}^{T}(0) \mathbf{J} \mathbf{g}_{1}^{*}(0) \\
\mathbf{g}_{2}^{H}(0) \mathbf{J} \mathbf{g}_{2}(0) & 0 & \mathbf{g}_{1}^{H}(0) \mathbf{J} \mathbf{g}_{1}(0) & 0
\end{array}\right], }
\end{aligned}
$$

$$
\begin{aligned}
\mathbf{C}_{2}= & {\left[\begin{array}{cccc}
\mathbf{g}_{1}^{T}(0) \mathbf{h}_{1}(0) & 0 & \mathbf{g}_{2}^{T}(0) \mathbf{h}_{2}(0) & 0 \\
0 & \mathbf{g}_{1}^{T}(0) \mathbf{h}_{1}(0) & 0 & \mathbf{g}_{2}^{T}(0) \mathbf{h}_{2}(0) \\
\mathbf{g}_{2}^{T}(0) \mathbf{h}_{2(0)} & 0 & \mathbf{g}_{1}^{T}(0) \mathbf{h}_{1}(0) & 0 \\
0 & \mathbf{g}_{2}^{T}(0) \mathbf{h}_{2}(0) & 0 & \mathbf{g}_{1}^{T}(0) \mathbf{h}_{1}(0)
\end{array}\right], } \\
\mathbf{D}_{2}= & {\left[\begin{array}{cccc}
0 & \mathbf{g}_{1}^{T}(0) \mathbf{J} \mathbf{h}_{1}^{*}(0) & 0 & \mathbf{g}_{2}^{T}(0) \mathbf{J} \mathbf{h}_{2}^{*}(0) \\
\mathbf{h}_{1}^{H}(0) \mathbf{J} \mathbf{g}_{1}(0) & 0 & \mathbf{h}_{2}^{H}(0) \mathbf{J} \mathbf{g}_{2}(0) & 0 \\
0 & \mathbf{g}_{2}^{T}(0) \mathbf{J} \mathbf{h}_{2}^{*}(0) & 0 & \mathbf{g}_{1}^{T}(0) \mathbf{J} \mathbf{h}_{1}^{*}(0) \\
\mathbf{h}_{2}^{H}(0) \mathbf{J} \mathbf{g}_{2}(0) & 0 & \mathbf{h}_{1}^{H}(0) \mathbf{J} \mathbf{g}_{1}(0) & 0
\end{array}\right], }
\end{aligned}
$$

and

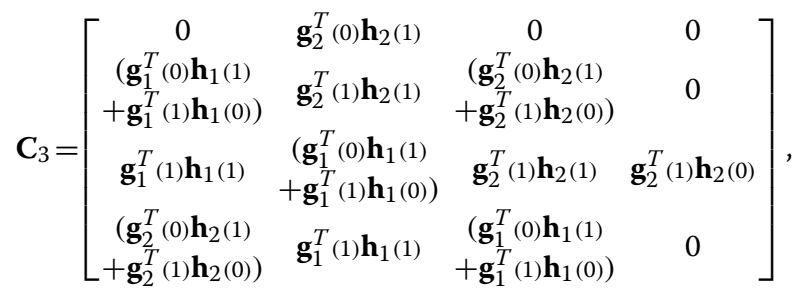

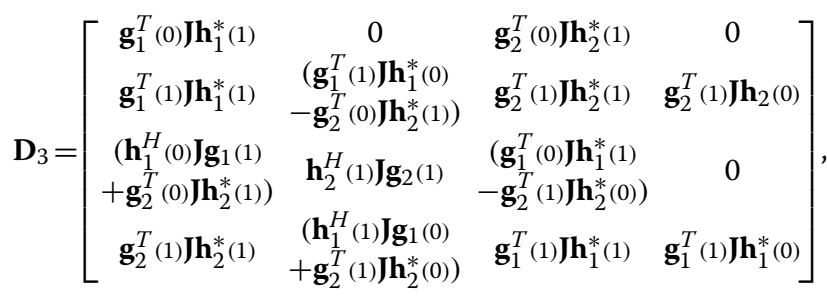

where $\mathbf{J}=\left[\begin{array}{cc}0 & 1 \\ -1 & 0\end{array}\right]$. The values of $\mathbf{q}_{1, x}, \mathbf{q}_{1, y}$ and $\mathbf{q}_{0, x}$ are given by

$$
\begin{aligned}
& \underset{4 \times 1}{\mathbf{q}_{1, x}(i)} \\
& =\left[\begin{array}{c}
\left(2 \mathbf{g}_{1}^{T}(0) \mathbf{g}_{1}(0) \mathbf{x}(i-1,4)+\mathbf{g}_{2}^{T}(0) \mathbf{g}_{2}(1) \mathbf{x}(i-1,2)-\mathbf{g}_{1}^{T}(1) \mathbf{J} \mathbf{g}_{1}^{*}(0) \mathbf{x}^{*}(i-1,3)\right. \\
\left.-\mathbf{g}_{2}^{T}(1) \mathbf{J} \mathbf{g}_{2}^{*}(0) \mathbf{x}^{*}(i-1,1)-\mathbf{g}_{2}^{T}(1) \mathbf{g} \mathbf{g}_{2}^{*}(1) \mathbf{x}^{*}(i-2,4)\right) \\
-\mathbf{g}_{1}^{T}(0) \mathbf{g} \mathbf{g}_{1}^{*}(1) \mathbf{x}^{*}(i-1,4) \\
\mathbf{g}_{2}^{T}(0) \mathbf{g}_{2}(1) \mathbf{x}(i-1,4) \\
-\mathbf{g}_{2}^{T}(0) \mathbf{g} \mathbf{g}_{2}^{*}(1) \mathbf{x}^{*}(i-1,4)
\end{array}\right],
\end{aligned}
$$


$\underset{4 \times 1}{\mathbf{q}_{1, y}(i)}$

$$
=\left[\begin{array}{c}
\left(\mathbf{g}_{2}^{T}(1) \mathbf{h}_{2}(0) \mathbf{x}(i-1,2)-\mathbf{g}_{1}^{T}(1) \mathbf{J} \mathbf{h}_{1}^{*}(0) \mathbf{x}^{*}(i-1,3)-\mathbf{g}_{2}^{T}(1)\right) \mathbf{h}_{2}^{*}(0) \mathbf{x}^{*}(i-1,1) \\
\left.-\mathbf{g}_{2}^{T}(1) \mathbf{h} \mathbf{h}_{2}^{*}(1) \mathbf{x}^{*}(i-2,4)+\left(\mathbf{g}_{2}^{T}(0) \mathbf{h}_{1}(1)+\mathbf{g}_{1}^{T}(0) \mathbf{h}_{1(1)}\right) \mathbf{x}(i-1,4)\right) \\
\left.-\mathbf{g}_{1}^{T}(0)\right) \mathbf{h}_{1}^{*}(1) \mathbf{x}^{*}(i-1,4) \\
\mathbf{g}_{2}^{T}(0) \mathbf{h}_{2}(1) \mathbf{x}(i-1,4) \\
\left.-\mathbf{g}_{2}^{T}(0)\right) \mathbf{h}_{2}^{*}(1) \mathbf{x}^{*}(i-1,4)
\end{array}\right]
$$

and $\mathbf{q}_{0, x}(i)=\left[\mathbf{q}_{0, x}(i, 1), \mathbf{q}_{0, x}(i, 2), \mathbf{q}_{0, x}(i, 3), \mathbf{q}_{0, x}(i, 4)\right]^{T}$, respectively, where

$$
\begin{aligned}
\mathbf{q}_{0, x}(i, 1)= & \mathbf{g}_{2}^{T}(0) \\
+ & \mathbf{g}_{1}(1) \mathbf{x}(i, 2)+\mathbf{g}_{1}^{T}(0) \mathbf{J} \mathbf{J g}_{1}^{*}(1) \mathbf{x}^{*}(i, 1) \mathbf{x}^{*}(i, 3) \\
\mathbf{q}_{0, x}(i, 2)= & 2 \mathbf{g}_{1}^{T}(0) \mathbf{g}_{1}(1) \mathbf{x}(i, 1)+2 \mathbf{g}_{2}^{T}(0) \mathbf{g}_{2}(1) \mathbf{x}(i, 3) \\
& +\mathbf{g}_{2}^{T}(1) \mathbf{J} \mathbf{g}_{2}^{*}(0) \mathbf{x}^{*}(i, 4)+\mathbf{g}_{1}^{T}(1) \mathbf{J} \mathbf{g}_{1}^{*}(1) \mathbf{x}^{*}(i, 1) \\
& +\mathbf{g}_{2}^{T}(1) \mathbf{J} \mathbf{g}_{2}^{*}(1) \mathbf{x}^{*}(i, 3)+\mathbf{g}_{2}^{T}(1) \mathbf{g}_{2}(1) \mathbf{x}(i, 2) \\
& +\left(\mathbf{g}_{1}^{T}(1) \mathbf{J} \mathbf{g}_{1}^{*}(0)-\mathbf{g}_{2}^{T}(0) \mathbf{J} \mathbf{g}_{2}^{*}(1)\right) \mathbf{x}^{*}(i, 2),
\end{aligned}
$$

$$
\begin{aligned}
\mathbf{q}_{0, x}(i, 3)= & 2 \mathbf{g}_{1}^{T}(0) \mathbf{g}_{1}(1) \mathbf{x}(i, 2)+\mathbf{g}_{2}^{T}(0) \mathbf{g}_{2}(1) \mathbf{x}(i, 4) \\
& -\mathbf{g}_{2}^{T}(1) \mathbf{J} \mathbf{g}_{2}^{*}(1) \mathbf{x}^{*}(i, 2)+\mathbf{g}_{1}^{T}(1) \mathbf{g}_{1}(1) \mathbf{x}(i, 1) \\
& +\mathbf{g}_{2}^{T}(1) \mathbf{g}_{2}(1) \mathbf{x}(i, 3)-\left(\mathbf{g}_{1}^{T}(1) \mathbf{J} \mathbf{g}_{1}^{*}(0)\right. \\
& \left.-\mathbf{g}_{2}^{T}(0) \mathbf{J} \mathbf{g}_{2}^{*}(1)\right) \mathbf{x}^{*}(i, 2)+\left(\mathbf{g}_{1}^{T}(0) \mathbf{J} \mathbf{g}_{1}^{*}(1)\right. \\
& \left.-\mathbf{g}_{2}^{T}{ }^{(1)} \mathbf{J} \mathbf{g}_{2}^{*}(0)\right) \mathbf{x}^{*}(i, 3)
\end{aligned}
$$

and

$$
\begin{aligned}
\mathbf{q}_{0, x}(i, 4)= & 2 \mathbf{g}_{1}^{T}{ }^{(0)} \mathbf{g}_{1}(1) \mathbf{x}(i, 3)+2 \mathbf{g}_{2}^{T}(0) \mathbf{g}_{2}(1) \mathbf{x}(i, 1) \\
& +\mathbf{g}_{1}^{T}{ }_{(1)} \mathbf{J} \mathbf{g}_{1}^{*}(0) \mathbf{x}^{*}(i, 4)+\mathbf{g}_{1}^{T}(1) \mathbf{J g}_{1}^{*}(1) \mathbf{x}^{*}(i, 3) \\
& +\mathbf{g}_{2}^{T}{ }_{(1)} \mathbf{J} \mathbf{g}_{2}^{*}(1) \mathbf{x}^{*}(i, 1)+\mathbf{g}_{1}^{T}(1) \mathbf{g}_{1}(1) \mathbf{x}(i, 2) \\
& -\left(\mathbf{g}_{1}^{T}(0) \mathbf{J} \mathbf{g}_{1}^{*}(1)-\mathbf{g}_{2}^{T}(1) \mathbf{J} \mathbf{g}_{2}^{*}(0)\right) \mathbf{x}^{*}(i, 2)
\end{aligned}
$$

\subsection{Two-relay TWRNs using Alamouti's STBC}

Another instance is shown here for a case of two singleantenna relay TWRNs, meaning that $R_{a}=1$ and $N=2$. This network uses Alamouti's STBC that is proposed in [19]. The encoding matrices used by these relays in this network are shown in [20]. This is an orthogonal STBC and full-rate code. The data exchanged through the net- work is $\mathbf{x}(i)=[\mathbf{x}(i, 1), \mathbf{x}(i, 2)]^{T}$ and $\mathbf{y}(i)=[\mathbf{y}(i, 1), \mathbf{y}(i, 2)]^{T}$. The instance model can be represented as

$$
\begin{aligned}
& \mathbf{C}_{1}=\left[\begin{array}{cc}
g_{1}^{2}(0) & 0 \\
0 & g_{1}^{2}(0)
\end{array}\right], \mathbf{D}_{1}=\left[\begin{array}{cc}
0 & -\left|g_{2}\right|^{2}(0) \\
\left|g_{2}\right|^{2}(0) & 0
\end{array}\right], \\
& \mathbf{C}_{2}=\left[\begin{array}{cc}
g_{1}(0) h_{1}(0) & 0 \\
0 & g_{1}(0) h_{1}(0)
\end{array}\right], \mathbf{D}_{2}=\left[\begin{array}{cc}
0 & -g_{2}(0) h_{2}^{*}(0) \\
g_{2}(0) h_{2}^{*}(0) & 0
\end{array}\right],
\end{aligned}
$$

and

$$
\begin{aligned}
& \mathbf{C}_{3}=\left[\begin{array}{cc}
0 & 0 \\
g_{1}(0) h_{1}(1)+g_{1}(1) h_{1}(0) & 0
\end{array}\right], \\
& \mathbf{D}_{3}=\left[\begin{array}{cc}
-g_{2}(0) h_{2}^{*}(1) & 0 \\
-g_{2}(1) h_{2}^{*}(1) & -g_{2}(1) h_{2}^{*}(0)
\end{array}\right] .
\end{aligned}
$$

The values of $\mathbf{q}_{1, x}, \mathbf{q}_{1, y}$ and $\mathbf{q}_{0, x}$ are given by

$$
\underset{2 \times 1}{\mathbf{q}_{1, x}(i)}=\left[\begin{array}{c}
\left(\left|g_{2}(1)\right|^{2} \mathbf{x}^{*}(i-2,2)+g_{2}(1) g_{2}^{*}(0) \mathbf{x}^{*}(i-1,1)\right. \\
\left.+2 g_{1}(0) g_{1}(1) \mathbf{x}(i-1,2)\right) \\
g_{2}(0) g_{2}^{*}(1) \mathbf{x}^{*}(i-1,2)
\end{array}\right],
$$

$\underset{2 \times 1}{\mathbf{q}_{1, y}}(i)=\left[\begin{array}{c}\left(\left(g_{1}(0) h_{1}(1)+g_{1}(1) h_{1}(0)\right) \mathbf{x}(i-1,2)\right. \\ \left.+g_{2}(1) h_{2}(0) \mathbf{x}^{*}(i-1,1)+g_{2}(1) h_{2}(1) \mathbf{x}^{*}(i-2,2)\right) \\ g_{2}(0) h_{2}^{*}(1) \mathbf{x}^{*}(i-1,2)\end{array}\right]$,

and

$$
\underset{2 \times 1}{\mathbf{q}_{0, x}(i)}=\left[\begin{array}{c}
-g_{2}(0) g_{2}^{*}(1) \mathbf{x}^{*}(i, 1) \\
\left(2 g_{1}(0) g_{1}(1) \mathbf{x}(i, 1)-g_{2}(1) g_{2}^{*}(0) \mathbf{x}^{*}(i, 2)\right. \\
\left.-\left|g_{2}(1)\right|^{2} \mathbf{x}^{*}(i, 1)\right)
\end{array}\right] .
$$

\subsection{Four-relay TWRNs using QO-STBC}

A TWRN with four single-antenna relays that use QOSTBC from [20] is evaluated, meaning that $R_{a}=1, N=4$ and $J=4$. This network along with the encoding matrices is shown in [9]. The instance model can be represented as

$$
\begin{aligned}
\mathbf{C}_{1}= & {\left[\begin{array}{cccc}
g_{1}^{2}(0) & 0 & 0 & g_{4}^{2}(0) \\
0 & g_{1}^{2}(0) & -g_{4}^{2}(0) & 0 \\
0 & -g_{4}^{2}(0) & g_{1}^{2}(0) & 0 \\
g_{4}^{2}(0) & 0 & 0 & g_{1}^{2}(0)
\end{array}\right], } \\
\mathbf{D}_{1}= & {\left[\begin{array}{cccc}
0 & -\left|g_{2}(0)\right|^{2} & -\left|g_{3}(0)\right|^{2} & 0 \\
\left|g_{2}(0)\right|^{2} & 0 & 0 & -\left|g_{3}(0)\right|^{2} \\
\left|g_{3}(0)\right|^{2} & 0 & 0 & -\left|g_{2}(0)\right|^{2} \\
0 & \left|g_{3}(0)\right|^{2} & \left|g_{2}(0)\right|^{2} & 0
\end{array}\right], }
\end{aligned}
$$




$$
\begin{aligned}
\mathbf{C}_{2} & =\left[\begin{array}{cccc}
g_{1}(0) h_{1}(0) & 0 & 0 & g_{4}(0) h_{4}(0) \\
0 & g_{1}(0) h_{1}(0) & -g_{4}(0) h_{4}(0) & 0 \\
0 & -g_{4}(0) h_{4}(0) & g_{1}(0) h_{1}(0) & 0 \\
g_{4}(0) h_{4}(0) & 0 & 0 & g_{1}(0) h_{1}(0)
\end{array}\right], \\
\mathbf{D}_{2} & =\left[\begin{array}{cccc}
0 & -g_{2}(0) h_{2}^{*}(0) & -g_{3}(0) h_{3}^{*}(0) & 0 \\
g_{2}(0) h_{2}^{*}(0) & 0 & 0 & -g_{3}(0) h_{3}^{*}(0) \\
g_{3}(0) h_{3}^{*}(0) & 0 & 0 & -g_{2}(0) h_{2}^{*}(0) \\
0 & g_{3}(0) h_{3}^{*}(0) & g_{2}(0) h_{2}^{*}(0) & 0
\end{array}\right],
\end{aligned}
$$

and

$\mathbf{C}_{3}=\left[\begin{array}{cccc}0 & 0 & g_{4}(0) h_{4}(1) & 0 \\ \left(g_{1(0)} h_{1(1)}\right. & -g_{4}(0) h_{4}(1) & g_{4}(1) h_{4}(1) & g_{4}(1) h_{4}(0) \\ \left.+g_{1(1)} h_{1}(0)\right) & \left(g_{1}(0) h_{1}(1)\right. & & \\ \left(g_{1(1)} h_{1(1)}\right. & +g_{1(1)} h_{1}(0) & -g_{4}(1) h_{4}(0) & 0 \\ \left.-g_{4}(0) h_{4}(1)\right) & \left.-g_{4}(1) h_{4}(1)\right) & & \\ -g_{4}(1) h_{4}(1) & \left(g_{1}(1) h_{1}(1)\right. & \left(g_{1}(0) h_{1}(1)\right. & 0\end{array}\right]$

$\mathbf{D}_{3}=\left[\begin{array}{cccc}-g_{2}(0) h_{2}^{*}(1) & -g_{3}(0) h_{3}^{*}(1) & 0 & 0 \\ -g_{2}(1) h_{2}^{*}(1) & \left(-g_{2}(1) h_{2}^{*}(0)\right. & \left(-g_{3}(1) h_{3}^{*}(1)\right. & 0 \\ & \left.-g_{3}(1) h_{3}^{*}(1)\right) & \left.-g_{3}(1) h_{3}^{*}(0)\right) & \\ g_{2}(1) h_{2}^{*}(0) & 0 & \left(-g_{2}(0) h_{2}^{*}(1)\right. & -g_{3}(1) h_{3}^{*}(0) \\ & 0 & \left.-g_{3}(1) h_{1}^{*}(1)\right) & \\ g_{3}(0) h_{3}^{*}(1) & \left(g_{3}(0) h_{3}^{*}(1)\right. & -g_{2}(0) h_{2}^{*}(1) & -g_{2}(1) h_{2}^{*}(0)\end{array}\right]$.

The values of $\mathbf{q}_{1, x}, \mathbf{q}_{1, y}$ and $\mathbf{q}_{0, x}$ are given by

$$
\mathbf{q}_{1, x}(i)=\left[\begin{array}{c}
\left(g_{4}^{2}(1) \mathbf{x}(i-2,4)+g_{4}(0) g_{4}(1) \mathbf{x}(i-1,1)+g_{2}(1) g_{2}^{*}(0) \mathbf{x}^{*}(i-1,3)\right. \\
\left.+g_{3}(1) g_{3}^{*}(0) \mathbf{x}^{*}(i-1,2)+2 g_{1}(0) g_{1}(1) x(i-1,4)\right) \\
g_{2(0)} g_{2}^{*}(1) \mathbf{x}^{*}(i-1,4) \\
g_{3(0)} g_{3}^{*}(1) \mathbf{x}^{*}(i-1,4) \\
g_{4(0)} g_{4(1)} \mathbf{x}(i-1,4)
\end{array}\right],
$$

$\mathbf{q}_{1, y}(i)=\left[\begin{array}{c}\left(\left(g_{1}(0) h_{1}(1)+g_{1}(1) h_{1}(0)\right) \mathbf{x}(i-1,4)+g_{2}(1) h_{2}^{*}(0) \mathbf{x}^{*}(i-1,3)\right. \\ \left.+g_{3}(1) h_{3}^{*}(0) \mathbf{x}^{*}(i-1,2)+g_{4}(1) h_{4}(0) \mathbf{x}(i-1,1)+g_{4}(1) h_{4}(1) x(i-2,4)\right) \\ g_{2}(0) h_{2}^{*}(1) \mathbf{x}^{*}(i-1,4) \\ g_{3}(0) h_{3}^{*}(1) \mathbf{x}^{*}(i-1,4) \\ g_{4}(0) h_{4}(1) \mathbf{x}(i-1,4)\end{array}\right]$,

and $\mathbf{q}_{0, x}(i)=\left[\mathbf{q}_{0, x}(i, 1), \mathbf{q}_{0, x}(i, 2), \mathbf{q}_{0, x}(i, 3), \mathbf{q}_{0, x}(i, 4)\right]^{T}$, respectively, where

$$
\begin{aligned}
\mathbf{q}_{0, x}(i, 1)= & g_{4}(0) g_{4}(1) \mathbf{x}(i, 3)-g_{3}(0) g_{3}^{*}(1) \mathbf{x}^{*}(i, 2) \\
& -g_{2}(0) g_{2}^{*}(1) \mathbf{x}^{*}(i, 1),
\end{aligned}
$$

$$
\begin{aligned}
\mathbf{q}_{0, x}(i, 2)= & -\left|g_{2}(1)\right|^{2} \mathbf{x}^{*}(i, 1)-\left(\left|g_{3}(1)\right|^{2}+g_{2}(1) g_{2}^{*}(0)\right) \mathbf{x}^{*}(i, 2) \\
& -\left(g_{3}(0) g_{3}^{*}(1)+g_{3}(1) g_{3}^{*}(0)\right) \mathbf{x}^{*}(i, 3)+g_{4}^{2}(1) \mathbf{x}(i, 3) \\
& +2 g_{1}(0) g_{1}(1) \mathbf{x}(i, 1)-g_{4}(0) g_{4}(1) x(i, 2) \\
& +g_{4}(0) g_{4}(1) \mathbf{x}(i, 4),
\end{aligned}
$$

$\mathbf{q}_{0, x}(i, 3)=\left(-g_{4}^{2}(1)+2 g_{1}(0) g_{1}(1)\right) \mathbf{x}(i, 2)-\left(g_{4}(0) g_{4}(1)-g_{1}^{2}(1)\right)$

$$
\begin{aligned}
& \times \mathbf{x}(i, 1)+g_{2}(1) g_{2}^{*}(0) \mathbf{x}^{*}(i, 1)-\left(g_{2}(0) g_{2}^{*}(1)\right. \\
& \left.+g_{3}(1) g_{3}^{*}(1)\right) \mathbf{x}^{*}(i, 3)-g_{3}(1) g_{3}^{*}(0) \mathbf{x}^{*}(i, 4) \\
& -g_{4}(0) g_{4}(1) \mathbf{x}(i, 3)
\end{aligned}
$$

and

$$
\begin{aligned}
\mathbf{q}_{0, x}(i, 4)= & \left(g_{3}(0) g_{3}^{*}(1)+g_{3}(1) g_{3}^{*}(0)\right) \mathbf{x}^{*}(i, 1)-\left(g_{4}(0) g_{4}(1)-g_{1}^{2}(1)\right) \\
& \times \mathbf{x}(i, 2)+g_{2}(0) g_{2}^{*}(1) \mathbf{x}^{*}(i, 2)-g_{4}^{2}(1) \mathbf{x}(i, 1) \\
& -\left|g_{2}(1)\right|^{2} \mathbf{x}^{*}(i, 3)-g_{2}(1) g_{2}^{*}(0) \mathbf{x}^{*}(i, 4) \\
& +2 g_{1}(0) g_{1}(1) \mathbf{x}(i, 3) .
\end{aligned}
$$

\section{Detection methods}

In this section, the conventional detector is outlined, and then, the two existing detectors of $[11,12]$ are extended for TWRNs. In addition, an enhanced detector is proposed. Its performance outperforms that of the extended detectors.

\subsection{Conventional detector}

- Self-interference cancellation:

$$
\widetilde{\mathbf{z}}_{1}(i)=\overline{\mathbf{z}}_{1}(i)-\sqrt{P_{1}} \beta \mathbf{G}_{\mathbf{G}} \overline{\mathbf{x}}(i) .
$$

- Equalisation process: obtain the equalised vector $\mathbf{u}_{1}$ by

$$
\begin{aligned}
\mathbf{u}_{1}(i)= & \mathbf{G}_{\mathbf{H}}^{H} \widetilde{\bar{z}}_{1}(i)=\sqrt{P_{2}} \beta \mathcal{H} \overline{\mathbf{y}}(i) \\
& +\sqrt{P_{2}} \beta \mathcal{B} \overline{\mathbf{y}}+\mathbf{G}_{\mathbf{H}}^{H}\left(\overline{\mathbf{q}}_{1, y}+\overline{\mathbf{q}}_{0, x}+\overline{\mathbf{q}}_{1, x}\right)+\mathbf{G}_{\mathbf{H}}^{H} \bar{\zeta}_{1},
\end{aligned}
$$

where $\mathcal{H}=\mathbf{G}_{\mathbf{H}}^{H} \mathbf{G}_{\mathbf{H}}$ and $\mathcal{B}=\mathbf{G}_{\mathbf{H}}^{H} \mathbf{G}_{q}$.

- Group decoding: assuming a code with $L$ decoding groups, the decoding can be carried through (if the used code is fully orthogonal, then $L=J$, see [20]),

$$
\left.\widehat{\mathbf{y}}_{l}(i)=\underset{\mathbf{y}_{l} \in \mathcal{S}^{(J / L) \times 1}}{\operatorname{argmin}}|| \mathbf{d}_{l}(i)-\left.\mathcal{H}_{l} \overline{\mathbf{y}}_{l}\right|^{2}\right\}, \forall l=1, \ldots, L,
$$


where $\widehat{\mathbf{y}}_{l}$ is the vector contained the estimation result of symbol group $l$. Both $\mathbf{d}_{l}$ and $\mathcal{H}_{l}$ are the corresponding elements of the group $l$ of the $\mathbf{u}_{1}$ and $\mathcal{H}$, respectively. For example, for a code of Section 2.2 that has $J=4, L=2$, its indices are $\mathbf{s}_{1}=[1,3]$ and $\mathbf{s}_{2}=[2,4]$ for the first group and second group, respectively. Accordingly,

$$
\widehat{\mathbf{y}}(i)=\left[\widehat{\mathbf{y}}_{1}(i, 1), \widehat{\mathbf{y}}_{2}(i, 1), \widehat{\mathbf{y}}_{1}(i, 2), \widehat{\mathbf{y}}_{2}(i, 2)\right]^{T},
$$

$$
\begin{aligned}
& \mathbf{d}_{1}(i)=\left[\begin{array}{l}
\mathbf{u}_{1}(i, 1) \\
\mathbf{u}_{1}(i, 3)
\end{array}\right]+1 i\left[\begin{array}{l}
\mathbf{u}_{1}(i, 5) \\
\mathbf{u}_{1}(i, 7)
\end{array}\right], \\
& \mathbf{d}_{2}(i)=\left[\begin{array}{l}
\mathbf{u}_{1}(i, 2) \\
\mathbf{u}_{1}(i, 4)
\end{array}\right]+1 i\left[\begin{array}{l}
\mathbf{u}_{1}(i, 6) \\
\mathbf{u}_{1}(i, 8)
\end{array}\right],
\end{aligned}
$$

$$
\mathcal{H}_{1}=\left[\begin{array}{llll}
h_{11} & h_{12} & h_{13} & h_{14} \\
h_{31} & h_{32} & h_{33} & h_{34} \\
h_{51} & h_{52} & h_{53} & h_{54} \\
h_{71} & h_{72} & h_{73} & h_{74}
\end{array}\right], \mathcal{H}_{2}=\left[\begin{array}{llll}
h_{21} & h_{22} & h_{23} & h_{24} \\
h_{41} & h_{42} & h_{43} & h_{44} \\
h_{61} & h_{62} & h_{53} & h_{64} \\
h_{81} & h_{82} & h_{83} & h_{84}
\end{array}\right]
$$

as $\mathcal{H}$ for this code is modelled as

$$
\mathcal{H}=\left[\begin{array}{cccccccc}
h_{11} & 0 & h_{12} & 0 & h_{13} & 0 & h_{14} & 0 \\
0 & h_{21} & 0 & h_{22} & 0 & h_{23} & 0 & h_{24} \\
h_{31} & 0 & h_{32} & 0 & h_{33} & 0 & h_{34} & 0 \\
0 & h_{41} & 0 & h_{42} & 0 & h_{43} & 0 & h_{44} \\
h_{51} & 0 & h_{52} & 0 & h_{53} & 0 & h_{54} & 0 \\
0 & h_{61} & 0 & h_{62} & 0 & h_{63} & 0 & h_{64} \\
h_{71} & 0 & h_{72} & 0 & h_{73} & 0 & h_{74} & 0 \\
0 & h_{81} & 0 & h_{82} & 0 & h_{83} & 0 & h_{84}
\end{array}\right] .
$$

Due to the interference components in (43), the designed code matrix is compromised. Unless an interference cancellation is used, a significant performance degradation is introduced.

\subsection{Existing IC detectors}

As mentioned, the PIC- and SIC-based detectors are proposed for one-way WRNs [11,12]. Here, these detectors are extended for TWRNs. Unlike one-way networks, the interference that arises due to the imperfect synchronisation in TWRNs originates from two sources and is introduced in the two phases of transmission. The terms, from the current processing terminals, are known to the terminal, and hence, both $\mathbf{q}_{0, x}(i)$ and $\mathbf{q}_{1, x}(i)$ can be eliminated completely. In addition, if the transmission has been initialised properly, $\mathbf{q}_{1, y}(i)$ can be cancelled as well. This can be conducted through the modified version of the self-interference cancellation step of Section 3.1 as
- Self-interference cancellation:

$$
\begin{aligned}
\widetilde{\mathbf{z}}_{1}^{0} & =\widetilde{\mathbf{z}}_{1}-\overline{\mathbf{q}}_{0, x}-\overline{\mathbf{q}}_{1, x}-\overline{\mathbf{q}}_{1, y} \\
& =\sqrt{P_{2}} \beta \mathbf{G}_{\mathbf{H}} \overline{\mathbf{y}}+\overline{\mathbf{q}}_{0, y}+\bar{\zeta}_{1} .
\end{aligned}
$$

Most IC detectors differ in how they deal with the current-block interference term $\left(\mathbf{q}_{0, y}\right)$. However, they are all reliant on initialisation of the interference cancellation process by the DT detected symbols and feeding of the results into a new iteration of the cancellation and decoding process [12]. In addition to the mentioned difference between the one-way and two-way networks, the DT link is not commonly feasible in the TWRNs as it requires full-duplex terminals.

\subsubsection{Extended PIC-based detector}

Here, the PIC-based detector of [12] proposed for oneway WRNs is extended for TWRNs to combat the performance degradation due to the imperfect synchronisation. In [21], the PIC-based detector is used for TWRNs with only two relays that employ Alamouti's orthogonal STBC code. In contrast, the PIC-based detector is extended here for the TWRN with $N R_{a}$-antenna relays and can employ either an orthogonal or quasi-orthogonal STBC. The extended PIC can be conducted through

\section{Algorithm 1 Extended PIC-based detector for TWRNs 1. Set $k=0$. \\ 2. Initialise $\mathbf{y}^{(k)}$ using the DT detection results $\widehat{\mathbf{y}}_{\mathrm{DT}}$; if the DT link exists, see (4) and (5). Otherwise, set $\mathbf{y}^{(k)}$ using the result of the conventional detector that is shown in Section 3.1 applied to $\widetilde{\mathbf{z}}_{1}^{0}$ of (45).}

3. Set $k=k+1$ and remove more ISI by calculating $\widetilde{\mathbf{z}}_{1}^{0,(k)}=\widetilde{\mathbf{z}}_{1}^{0}-\overline{\mathbf{q}}_{0, y}^{(k-1)}$, where $\mathbf{q}_{0, y}^{(k-1)}$ is evaluated through (12) using the current value of $\mathbf{y}^{(k-1)}$.

4. Update $\mathbf{y}^{(k)}$ using the result of the conventional detector of Section 3.1 applied to $\widetilde{\mathbf{z}}_{1}^{0,(k)}$.

5. Go back to step 3 until $k \geq K$.

\subsubsection{Extended SIC-based detector}

In [11], a SIC-based detector is proposed to combat the performance degradation in one-way WRNs due to the imperfect synchronisation. This detector is designed for a network with four relays that employ the decodeand-forward relaying protocol and the orthogonal STBC codes. It was shown that the SIC-based detector pro- 
posed converges faster than the existing PIC ones due to the successive behaviour of the interference cancellation process. In [8], a SIC-based detector is designed for TWRNs that employ Alamouti's orthogonal STBC. Similar to the former section, the SIC-based detector is extended for TWRNs with an arbitrary number of relays and can either use orthogonal STBC or quasi-orthogonal STBCs.

\section{Algorithm 2 Extended SIC-based detector for TWRN.}

1. Set $k=0$.

2. Initialise $\mathbf{y}^{(k)}$ using the DT detection results $\widehat{\mathbf{y}}_{\mathrm{DT}}$; if the DT link is exists, see (4) and (5). Otherwise, set $\mathbf{y}^{(k)}$ using the result of the conventional detector that is shown in Section 3.1 applied to $\widetilde{\overline{\mathbf{z}}}_{1}^{0}$ of (45).

3. Assuming a $L$-decoding groups STBC is employed, determine the detection order $\mathbf{w}$ by comparing $\left\|\mathcal{B}_{l}\right\|^{2}, \forall l=1, \ldots, L$. For example, for the network in Section 2.2, set $\mathbf{w}=[1,2]$ if $\left\|\mathcal{B}_{1}\right\|^{2} \leq\left\|\mathcal{B}_{2}\right\|^{2}$ and otherwise, set $\mathbf{w}=[2,1]$.

4. Set $k=k+1$ and $w$ value by the first value of the order vector $\mathbf{w}$.

5. Remove more ISI by calculating $\mathbf{u}^{0,(k+1)}=\mathbf{G}_{\mathbf{H}}^{H} \widetilde{\mathbf{z}}_{1}^{0}-\mathcal{B}\left(\overline{\mathbf{y}}^{(k)}\right)^{*}$ using the current available detected symbols $\mathbf{y}^{(k)}$.

6. Estimate the symbols within the group $w\left(\widehat{\overline{\mathbf{y}}}_{w}\right)$ using the conventional detector of Section 3.1 applied for $\overline{\mathbf{u}}_{w}$.

7. Update the corresponding symbols of the group $w$ in $\mathbf{y}^{(k)}$ by the result obtained in the previous step.

8. Set $w$ value by the next value of the order vector $\mathbf{w}$. Otherwise, set $w=0$.

9. If $w=0$ go to step 10 . Otherwise, go back to step 5 .

10. Go back to step 4 until $k \geq K$.

\subsection{Proposed detector}

The idea behind the new detector is firstly explained, and then, its steps are designed. Unlike the existing detectors, the proposed detector tries to cancel the terms that must be eliminated and tries to benefit from the other terms in an innovative manner. Therefore, the achieved performance is enhanced as the reliance on the first low-performance conventional detection result is reduced. The enhancements are particularly clear in the first iteration of the detection process.
The explanation is conducted for the network shown in Section 2.2. Rewriting (45) for illustration purposes as

$$
\widetilde{\mathbf{z}}_{1}^{0}(i)=\sqrt{P_{2}} \beta \mathbf{G}_{\mathbf{H}} \overline{\mathbf{y}}(i)+\sqrt{P_{2}} \beta \mathbf{G}_{\mathbf{q}} \overline{\mathbf{y}}(i)+\bar{\zeta}_{1}(i),
$$

and assuming that the second, fourth, sixth and eighth columns of $\mathbf{G}_{\mathbf{q}}$ are zeros, then the equalisation process $\mathbf{u}_{1}(i)=\mathbf{G}_{\mathbf{H}}^{H} \widetilde{\mathbf{z}}_{1}^{0}(i)$ leads to (ignoring non-important terms):

$$
\begin{aligned}
\mathbf{u}_{1}(i)= & {\left[\begin{array}{cccccccc}
h_{11} & 0 & h_{12} & 0 & h_{13} & 0 & h_{14} & 0 \\
0 & h_{21} & 0 & h_{22} & 0 & h_{23} & 0 & h_{24} \\
h_{31} & 0 & h_{32} & 0 & h_{33} & 0 & h_{34} & 0 \\
0 & h_{41} & 0 & h_{42} & 0 & h_{43} & 0 & h_{44} \\
h_{51} & 0 & h_{52} & 0 & h_{53} & 0 & h_{54} & 0 \\
0 & h_{61} & 0 & h_{62} & 0 & h_{63} & 0 & h_{64} \\
h_{71} & 0 & h_{72} & 0 & h_{73} & 0 & h_{74} & 0 \\
0 & h_{81} & 0 & h_{82} & 0 & h_{83} & 0 & h_{84}
\end{array}\right] \overline{\mathbf{y}}(i) } \\
& +\left[\begin{array}{cccccccc}
b_{11}^{1} & 0 & b_{12}^{1} & 0 & b_{13}^{1} & 0 & b_{14}^{1} & 0 \\
b_{21}^{1} & 0 & b_{22}^{1} & 0 & b_{23}^{1} & 0 & b_{24}^{1} & 0 \\
b_{31}^{1} & 0 & b_{32}^{1} & 0 & b_{33}^{1} & 0 & b_{34}^{1} & 0 \\
b_{41}^{1} & 0 & b_{42}^{1} & 0 & b_{43}^{1} & 0 & b_{44}^{1} & 0 \\
b_{51}^{1} & 0 & b_{52}^{1} & 0 & b_{53}^{1} & 0 & b_{54}^{1} & 0 \\
b_{61}^{1} & 0 & b_{62}^{1} & 0 & b_{63}^{1} & 0 & b_{64}^{1} & 0 \\
b_{71}^{1} & 0 & b_{72}^{1} & 0 & b_{73}^{1} & 0 & b_{74}^{1} & 0 \\
b_{81}^{1} & 0 & b_{82}^{1} & 0 & b_{83}^{1} & 0 & b_{84}^{1} & 0
\end{array}\right] \overline{\mathbf{y}}(i)
\end{aligned}
$$

Notice that all of the first, third, fifth and seventh time slots of $\mathbf{u}_{1}(i)$ are independent of $\mathbf{y}(i, 2)$ and $\mathbf{y}(i, 4)$. Therefore, the first group of symbols $\left(\mathbf{y}_{1}(i)=[\mathbf{y}(i, 1), \mathbf{y}(i, 3)]^{T}\right)$ can be detected independently through these mentioned slots of $\mathbf{u}_{1}(i)$, denoted as $\mathbf{u}_{1,1}(i)$. Similarly, the equalisation process provides

$$
\begin{aligned}
\mathbf{u}_{1}(i)= & {\left[\begin{array}{cccccccc}
h_{11} & 0 & h_{12} & 0 & h_{13} & 0 & h_{14} & 0 \\
0 & h_{21} & 0 & h_{22} & 0 & h_{23} & 0 & h_{24} \\
h_{31} & 0 & h_{32} & 0 & h_{33} & 0 & h_{34} & 0 \\
0 & h_{41} & 0 & h_{42} & 0 & h_{43} & 0 & h_{44} \\
h_{51} & 0 & h_{52} & 0 & h_{53} & 0 & h_{54} & 0 \\
0 & h_{61} & 0 & h_{62} & 0 & h_{63} & 0 & h_{64} \\
h_{71} & 0 & h_{72} & 0 & h_{73} & 0 & h_{74} & 0 \\
0 & h_{81} & 0 & h_{82} & 0 & h_{83} & 0 & h_{84}
\end{array}\right] \overline{\mathbf{y}}(i) } \\
& +\left[\begin{array}{ccccccccc}
0 & b_{11}^{2} & 0 & b_{12}^{2} & 0 & b_{13}^{2} & 0 & b_{14}^{2} \\
0 & b_{21}^{2} & 0 & b_{22}^{2} & 0 & b_{23}^{2} & 0 & b_{24}^{2} \\
0 & b_{31}^{2} & 0 & b_{32}^{2} & 0 & b_{33}^{2} & 0 & b_{34}^{2} \\
0 & b_{41}^{2} & 0 & b_{42}^{2} & 0 & b_{43}^{2} & 0 & b_{44}^{2} \\
0 & b_{51}^{2} & 0 & b_{52}^{2} & 0 & b_{53}^{2} & 0 & b_{54}^{2} \\
0 & b_{61}^{2} & 0 & b_{62}^{2} & 0 & b_{63}^{2} & 0 & b_{64}^{2} \\
0 & b_{71}^{2} & 0 & b_{72}^{2} & 0 & b_{73}^{2} & 0 & b_{74}^{2} \\
0 & b_{81}^{2} & 0 & b_{82}^{2} & 0 & b_{83}^{2} & 0 & b_{84}^{2}
\end{array}\right] \overline{\mathbf{y}}(i)
\end{aligned}
$$


if the first, third, fifth and seventh columns of $\mathbf{G}_{\mathbf{q}}$ are zeros. This leads to the second group of symbols $\left(\mathbf{y}_{2}(i)=[\mathbf{y}(i, 2), \mathbf{y}(i, 4)]^{T}\right)$ which can be detected independently through second, fourth, sixth and eighth time slots of $\mathbf{u}_{1}(i)$, denoted as $\mathbf{u}_{1,2}(i)$.

Assuming that the mentioned assumptions hold, the detection can be carried out for the two groups of symbol using

$$
\widehat{\mathbf{y}}_{l}(i)=\underset{\mathbf{y} l \in \mathcal{S}}{\operatorname{argmin}}\left|\overline{\mathbf{u}}_{1, l}-\left(\mathcal{H}_{l} \overline{\mathbf{y}}_{l}+\mathcal{B}_{l} \overline{\mathbf{y}}_{l}\right)\right|^{2},
$$

where $\mathcal{H}=\mathbf{G}_{\mathbf{H}}^{H} \mathbf{G}_{\mathbf{H}}$ and $\mathcal{B}=\mathbf{G}_{\mathbf{H}}^{H} \mathbf{G}_{\mathbf{q}} \cdot \mathcal{H}_{l}$ and $\mathcal{B}_{l}$ are the matrices that contained the corresponding elements of the group $l$ from $\mathcal{H}$ and $\mathcal{B}$, respectively. For example, for (46):

$\mathcal{H}_{1}(i)=\left[\begin{array}{llll}h_{11} & h_{12} & h_{13} & h_{14} \\ h_{31} & h_{32} & h_{33} & h_{34} \\ h_{51} & h_{52} & h_{53} & h_{54} \\ h_{71} & h_{72} & h_{73} & h_{74}\end{array}\right], \mathcal{B}_{1}=\left[\begin{array}{llll}b_{11}^{1} & b_{12}^{1} & b_{13}^{1} & b_{14}^{1} \\ b_{31}^{1} & b_{32}^{1} & b_{33}^{1} & b_{34}^{1} \\ b_{51}^{1} & b_{52}^{1} & b_{53}^{1} & b_{54}^{1} \\ b_{71}^{1} & b_{72}^{1} & b_{73}^{1} & b_{74}^{1}\end{array}\right]$,

and

$\mathcal{H}_{2}(i)=\left[\begin{array}{llll}h_{21} & h_{12} & h_{23} & h_{24} \\ h_{41} & h_{42} & h_{43} & h_{44} \\ h_{61} & h_{62} & h_{63} & h_{64} \\ h_{81} & h_{82} & h_{83} & h_{84}\end{array}\right], \mathcal{B}_{2}=\left[\begin{array}{llll}b_{21}^{2} & b_{22}^{2} & b_{23}^{2} & b_{24}^{2} \\ b_{41}^{2} & b_{42}^{2} & b_{43}^{2} & b_{44}^{2} \\ b_{61}^{2} & b_{62}^{2} & b_{63}^{2} & b_{64}^{2} \\ b_{81}^{2} & b_{82}^{2} & b_{83}^{2} & b_{84}^{2}\end{array}\right]$.

We now consider a method for cancelling these columns, and specifically, the order in which they are cancelled. Similar to all IC detectors, a subtraction of interference terms of the corresponding time slots is proposed. Logically, the ordering should be according to the ordering of reliability of available detected symbols. This

Table 1 The configurations of the simulated Networks

\begin{tabular}{lll}
\hline Denoted by & Used code & Network configurations \\
\hline Network 1 & Novel QO-STBC of [5] & $\begin{array}{l}\text { TWRN with two dual-antenna } \\
\text { relays; terminals are half-duplex } \\
\text { nodes. }\end{array}$ \\
\hline Network 2 & Novel QO-STBC of [5] & $\begin{array}{l}\text { TWRN with two dual-antenna } \\
\text { relays; terminals are full-duplex } \\
\text { nodes. }\end{array}$ \\
\hline Network 3 & Alamouti's STBC & $\begin{array}{l}\text { TWRN with two single-antenna } \\
\text { relays; terminals are half-duplex } \\
\text { nodes. }\end{array}$ \\
\hline Network 4 & QO-STBC of [9] & $\begin{array}{l}\text { TWRN with four single-antenna } \\
\text { relays; terminals are half-duplex } \\
\text { nodes. }\end{array}$ \\
& &
\end{tabular}

can be achieved by estimating which time slots have a better signal-to-noise ratio (SNR). The estimation of SNR values can be done through a two-phase training pilot sequence for channel estimation [22,23] and traditionally as in $[24,25]$ if the DT link exists. Consequently, better performance is achieved, particularly in the first iteration of the EIC detector, and hence, faster convergence is achieved in the consequent iteration.

\section{Algorithm 3 EIC-based detector for TWRNs.}

1. Set $k=0$ and initialise $\mathbf{y}^{(k)}$ using the DT detection results $\widehat{\mathbf{y}}_{\mathrm{DT}}$; if the DT link exists, see (4) and (5). Otherwise, set $\mathbf{y}^{(k)}$ using the result of the conventional detector that is shown in Section 3.1 applied to $\widetilde{\mathbf{z}}_{1}^{0}$ of (45).

2. Assuming a $L$-decoding groups $\mathrm{STBC}$ is employed, determine the detection order $\mathbf{w}$ by comparing the estimating values of the instantaneous SNRs for all $l=1, \ldots, L$. For example, for the network in Section 2.2, set $\mathbf{w}=[1,2,0]$ if $\gamma_{1} \leq \gamma_{2}$. Otherwise, set $\mathbf{w}=[2,1,0]$. If a DT link exists, the detection order of the first iteration is determined through the DT link symbols rather than the second phase symbols.

3. Set $k=k+1$ and $w$ value by the first value of the order vector $\mathbf{w}$.

4. For the current processing group $w$, remove more ISI by calculating

$$
\widetilde{\mathbf{z}}_{1}^{0,(k)}=\widetilde{\mathbf{z}}_{1}^{0}-\overline{\mathbf{q}}_{0, y}^{(k)},
$$

where $\mathbf{q}_{0, y}^{(k)}$ is evaluated through (12) using the previously detected symbols $\mathbf{y}^{(k-1)}$ and by setting the corresponding columns of the other groups of $\mathcal{B}$ to zeros. (For example, for the network in Section 2.2, these columns are the second, fourth, sixth and eighth columns if $w=1$ and the first, third, fifth and seventh columns when $w=2$ ).

5. Estimate the symbols of the current processing group through (49) using the last recent value of $\widetilde{\mathbf{z}}_{1}^{0,(k)}$, where $l=w$.

6. Update the corresponding symbols of the group $w$ in $\mathbf{y}^{(k)}$ by the result obtained in step 5 .

7. Set $w$ value by the next value of the order vector $\mathbf{w}$.

8. If $w=0$ go to step 10 . Otherwise, go back to step 5 .

9. Go back to step 4 until $k \geq K$. 


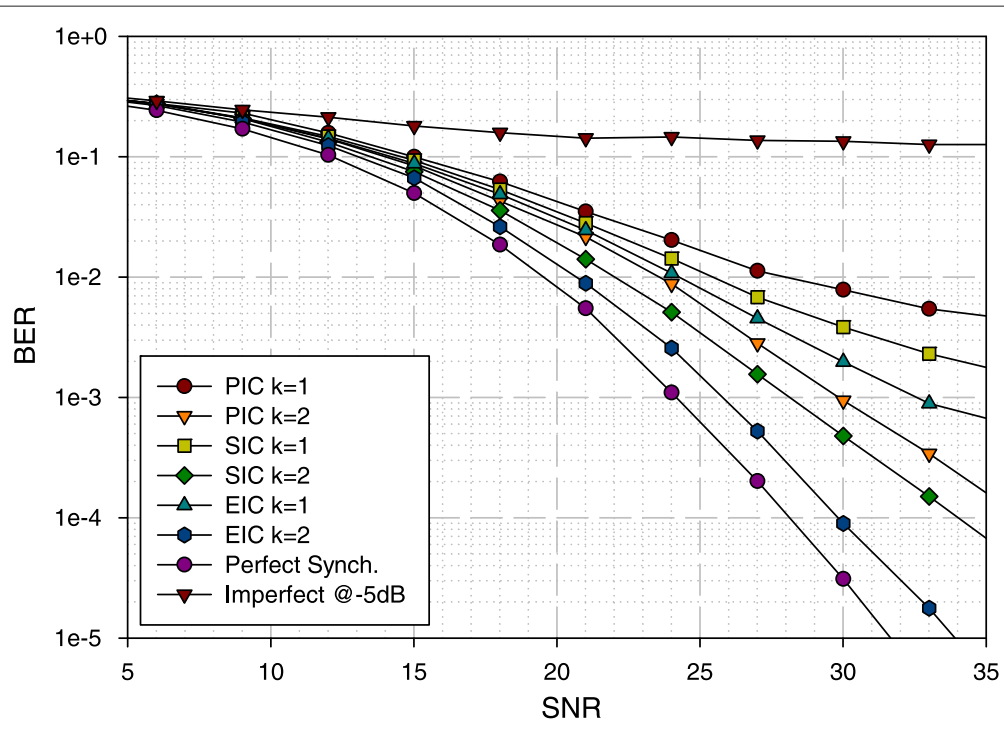

Figure 3 The BER using PIC, SIC and EIC detectors under a condition of $\alpha=-5 \mathrm{~dB}$ in Network 1.

\section{Simulation results and discussion}

In terms of bit error rate (BER), simulation results are presented to demonstrate the performance of the proposed detector under different conditions of imperfect synchronisation. Also, the results of both the extended PIC- and SIC-based detectors are included. The simulation uses 4-QAM Gray coded modulation over a Rayleigh fading channel. The configurations of simulated networks are shown in Table 1. The power loading scheme for each network is similar to the network under a condition of perfect synchronisation.
Network 1 is simulated under a condition of $\alpha=-5$ and $-3 \mathrm{~dB}$ in Figures 3 and 4, respectively. Figure 3 shows that even under a limited condition of imperfect synchronisation, much performance degradation is experienced if the conventional detector is used. This degradation can be mitigated if the extended PIC- and SIC-based detectors are used and better when the proposed EIC detector is used. Due to the innovative cancellation used, one iteration of EIC can offer a performance close to the performance of two iterations of either the PIC or SIC detector. In addition, it can offer a three

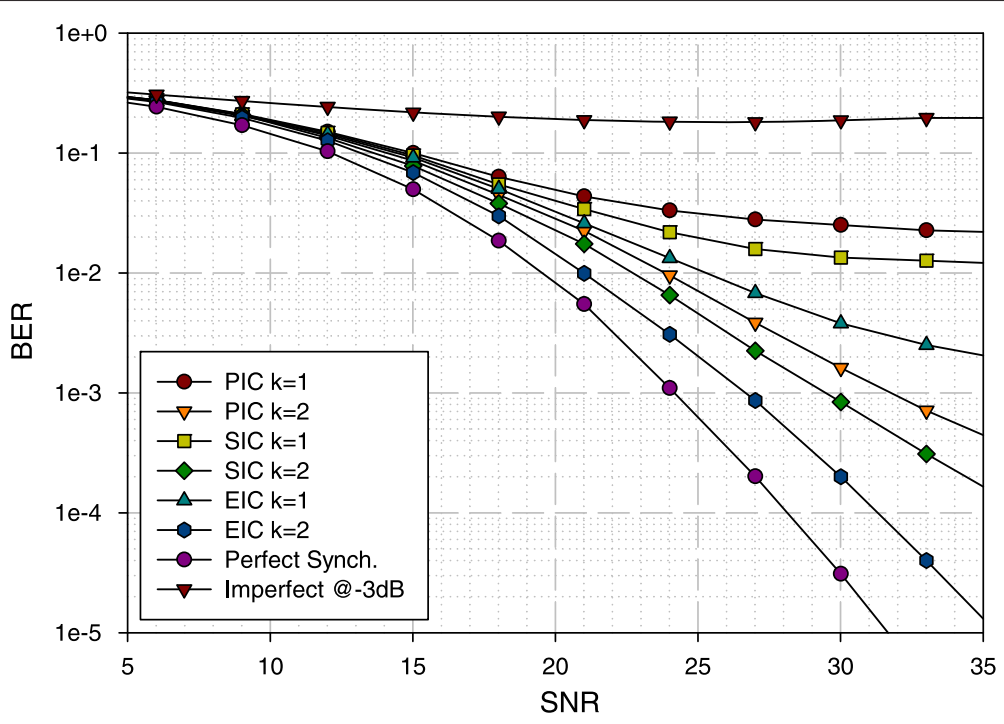

Figure 4 The BER using PIC, SIC and EIC detectors under a condition of $\alpha=-3 \mathrm{~dB}$ in Network 1. 


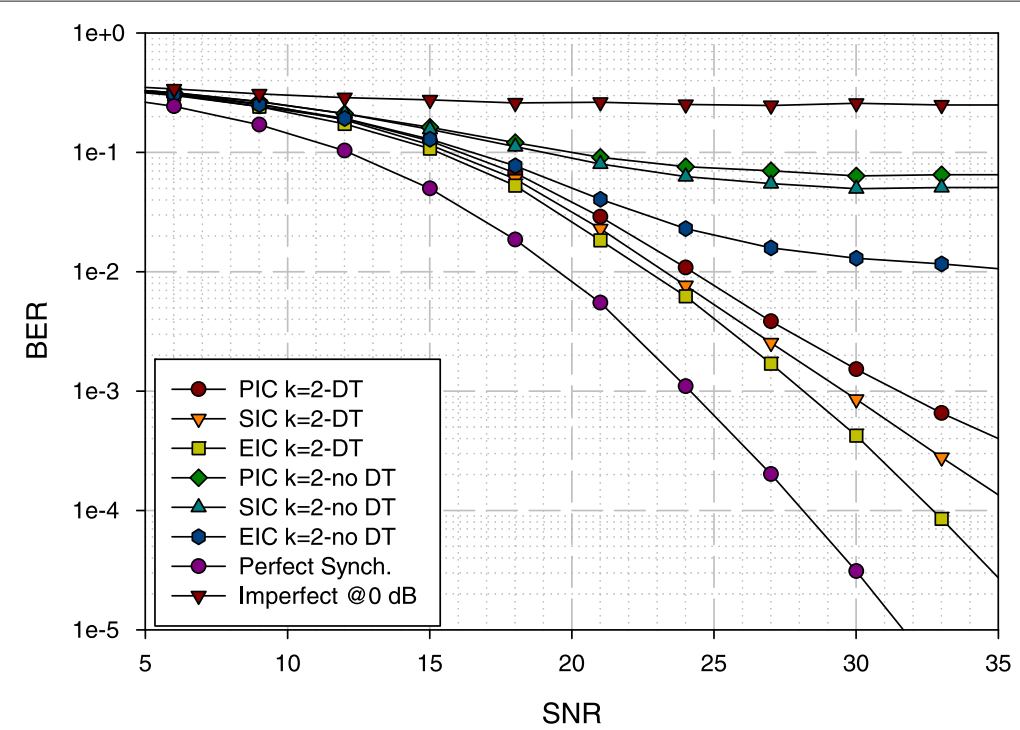

Figure 5 The BER using PIC, SIC and EIC detectors a condition of $\alpha=0 \mathrm{~dB}$ in Network 1 and Network 2.

to four SNR gain within two iterations to get a BER of $10^{-3}$ over the SIC and PIC detector, respectively. In Figure 4, the network is simulated under a condition of $-3 \mathrm{~dB}$ imperfect synchronisation. The performance of the extended detectors is degraded, but they are still effective compared to the conventional detector. In contrast, the EIC detector outperforms the extended detectors and shows a performance closer to the perfect synchronisation condition.

In Figure 5, the simulation results of Network 1 and Network 2 are included for the proposed and extended detector under a condition of $\alpha=0 \mathrm{~dB}$. This condition corresponds to the most severe value of time misalignment. In spite of severe timing errors, the proposed detector still outperforms the extended ones and performs much better than the conventional detector. It is worth noting that the EIC detector fails to achieve performance close to perfect synchronisation under this severe condition in the event of no DT link but it is still effective in the case where it exists.

The simulation results of using the proposed detectors in Network 3 and Network 4 are shown in Figures 6 and 7,

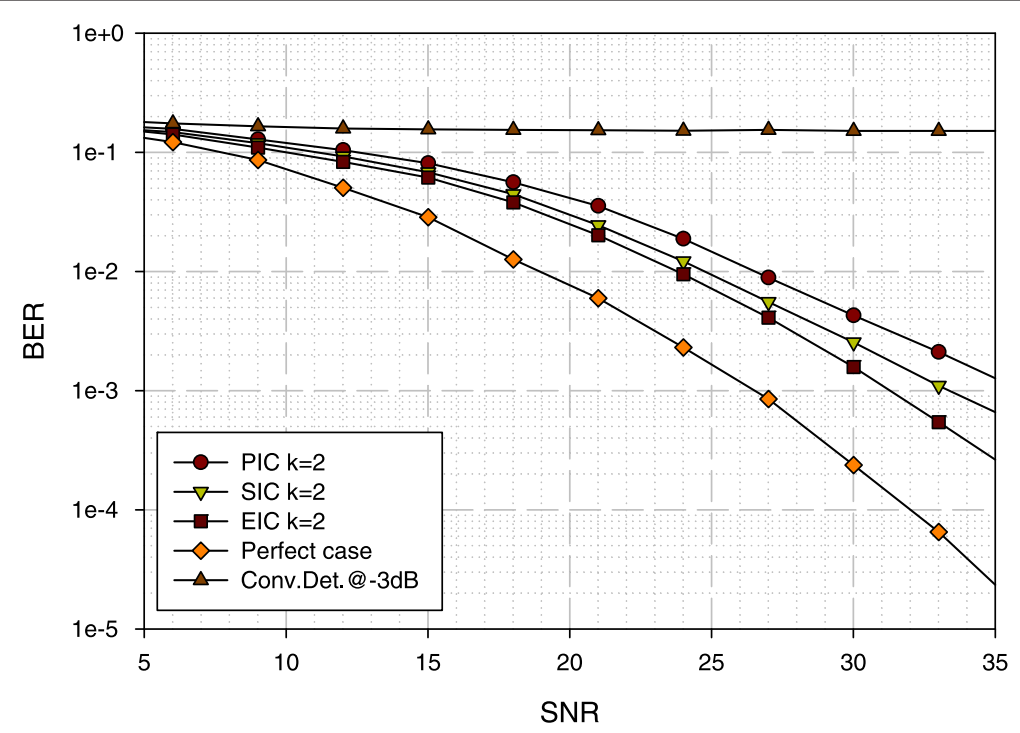

Figure 6 The BER using PIC, SIC and EIC detectors under a condition of $\alpha=-3 \mathrm{~dB}$ in Network 3 . 


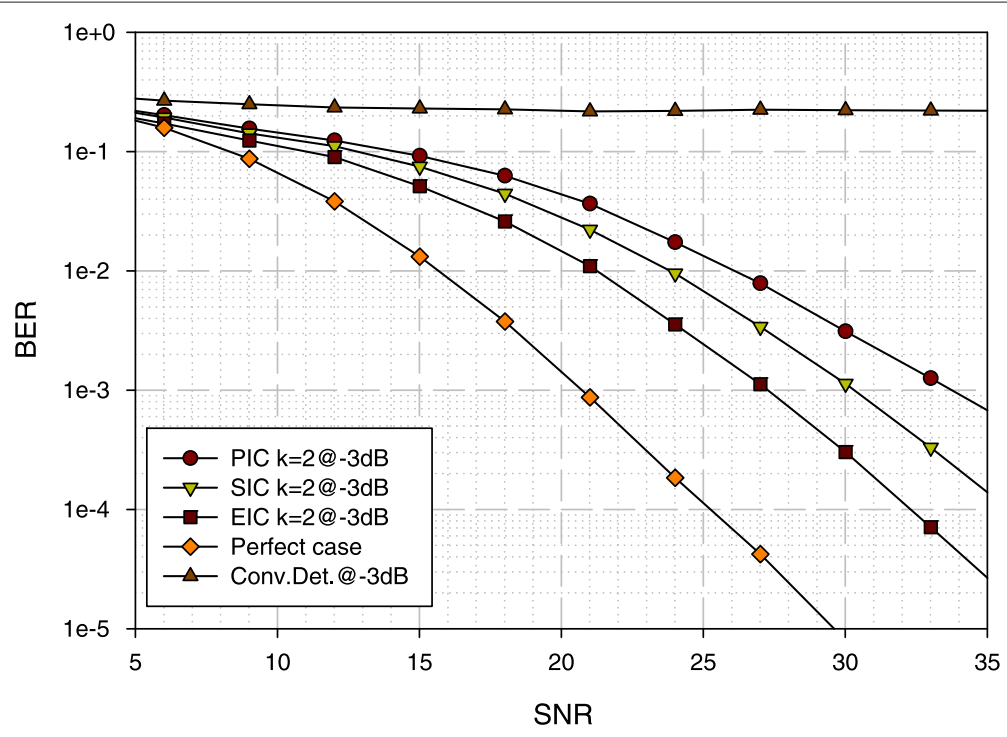

Figure 7 The BER using PIC, SIC and EIC detectors under a condition of $\alpha=-3 \mathrm{~dB}$ in Network 4.

respectively. They are simulated under a moderate condition of imperfect synchronisation, $\alpha=-3 \mathrm{~dB}$. The figures show that these detectors are designed not only for particular networks as in $[11,12]$ but also for any TWRNs with $N R_{a}$-antenna relays. In addition, they can be used for an orthogonal or quasi-orthogonal linear dispersion STBC. It can be concluded that the EIC detector outperforms the extended detectors and these extended detectors are still effective compared to the conventional detector.

\section{Conclusions}

A TWRN employing either quasi-orthogonal or orthogonal STBC was considered under imperfect synchronisation. The network consisted of two terminals exchanging data through $N R_{a}$-antenna relays. The relays employed the AF relaying protocol. The general model was derived for the considered network under a condition of imperfect synchronization, and then, instances were evaluated for the case of two single-antenna relays, two dual-antenna relays and four single-antenna relays. Both PIC- and SICbased detectors which were proposed previously for oneway relaying networks were extended here for TWRNs to mitigate the performance degradation due to the imperfect synchronisation. In addition, an EIC-based detector was proposed while maintaining low computational complexity. This detector showed better performance improvement as it is based on an innovative iterative cancellation process and it reduces the dependency on low-performance symbols. The simulation results show in terms of bit error rate that significant performance improvement can be gained compared to the conventional detector. Moreover, the proposed detector achieved better performance compared to the extended detectors under all conditions of imperfect synchronisation.

\section{Competing interests}

The authors declare that they have no competing interests.

Received: 21 May 2014 Accepted: 1 December 2014

Published: 6 December 2014

\section{References}

1. K Liu, A Sadek, S Weifeng, A Kwasinski, Cooperative Communications and Networking. (Cambridge University Press, London, UK, 2009)

2. JN Laneman, DN Tse, GW Wornell, Cooperative diversity in wireless networks: efficient protocols and outage behavior. IEEE Trans. Inf. Theory. 50(12), 3062-3080 (2004)

3. JN Laneman, GW Wornell, Distributed space-time-coded protocols for exploiting cooperative diversity in wireless networks. IEEE Trans. Inf. Theory. 49(10), 2415-2425 (2003)

4. T Cui, F Gao, T Ho, A Nallanathan, Distributed space-time coding for two-way wireless relay networks. IEEE Trans. Signal Process. 57(2), 658-671 (2009)

5. F Gong, J Zhang, J Ge, Novel distributed quasi-orthogonal space-time block codes for two-way two-antenna relay networks. IEEE Trans. Wireless Commun. 12(9), 4338-4349 (2013)

6. F Gong, J Zhang, J Ge, Distributed concatenated Alamouti codes for two-way relaying networks. IEEE Wireless Commun. Lett. 1(3), 197-200 (2012)

7. M Wang, F Wang, Z Zhong, Wireless MIMO switching: distributed zero-forcing and MMSE relaying using network coding. EURASIP J. Adv. Signal Process. 2013(1) (2013)

8. M.-T. O. El Astal, B.P. Salmon, J.C. Olivier, in Proceedings of the 2014 IEEE Wireless Communications and Networking Conference (WCNC). Distributed space-time block coding for two-way wireless relaying networks: improved performance under imperfect synchronization (IEEE Istanbul, Turkey, 2014), pp. 1176-1181

9. F Abdurahman, A Elazreg, JA Chambers, in Proceedings of the 20107 th International Symposium on Wireless Communication Systems (ISWCS). Distributed quasi-orthogonal space-time coding for two-way wireless relay networks (IEEE York, UK, 2010), pp. 413-416

10. S Wei, DL Goeckel, MC Valenti, Asynchronous cooperative diversity. IEEE Trans. on Wireless Commun. 5(6), 1547-1557 (2006) 
11. M-TO El Astal, AM Abu-Hudrouss, SIC detector for 4 relay distributed space-time block coding under quasi-synchronization. IEEE Commun. Lett. 15(10), 1056-1058 (2011)

12. F Zheng, AG Burr, S Olafsson, Signal detection for distributed space-time block coding: 4 relay nodes under quasi-synchronisation. IEEE Trans. Commun. 57(5), 1250-1255 (2009)

13. S Zhang, S-C Liew, PP Lam, in Procedding of the 2006 IEEE Information Theory Workshop (ITW). On the synchronization of physical-layer network coding (IEEE Chengdu, China, 2006), pp. 404-408

14. S Chang, B Kelley, An efficient time synchronization scheme for broadband two-way relaying networks based on physical-layer network coding. IEEE Commun. Lett. 16(9), 1416-1419 (2012)

15. Zhong Z, Zhu S, Nallanathan A, Distributed space-time trellis code for asynchronous cooperative communications under frequency-selective channels. IEEE Trans. Wireless Commun. 8(2), 796-805 (2009)

16. $Z \mathrm{Li}, \mathrm{XXia}, \mathrm{B} \mathrm{Li}$, Achieving full diversity and fast ML decoding via simple analog network coding for asynchronous two-way relay networks. IEEE Trans. Commun. 57(12), 3672-3681 (2009)

17. Y Liu, XXia, H Zhang, Distributed linear convolutional space-time coding for two-relay full-duplex asynchronous cooperative networks. IEEE Trans. Wireless Commun. 12(12), 6406-6417 (2013)

18. Z Zhong, S Zhu, G Lv, in Procedding of the 2009 IEEE International Conference on Communications. Distributed space-time code for asynchronous two-way wireless relay networks under frequency-selective channels (IEEE Dresden, Germany, 2009), pp. 1-5

19. SM Alamouti, A simple transmit diversity technique for wireless communications. IEEE J. Sel. Areas in Commun. 16(8), 1451-1458 (1998)

20. $\mathrm{Y}$ Jing, $\mathrm{H}$ Jafarkhani, Using orthogonal and quasi-orthogonal designs in wireless relay networks. IEEE Trans. Inf. Theory. 53(11), 4106-4118 (2007)

21. UN Mannai, FM Abdurahman, AM Elazreg, JA Chambers, in Proceeding of the 7th International Wireless Communications and Mobile Computing Conference (IWCMC). Orthogonal space time block coding for two-way wireless relay networks under imperfect synchronization (IEEE Istanbul, Turkey, 2011), pp. 1694-1697

22. S Zhang, F Gao, C Pei, Optimal training design for individual channel estimation in two-way relay networks. IEEE Trans. Signal Process. 60(9), 4987-4991 (2012)

23. $\mathrm{XXu}, \mathrm{J}$ Wu, S Ren, $\mathrm{X}$ Luan, $\mathrm{H}$ Xiang, in Proceeding of the International Conference on Advanced Communication Technology (ICACT). Superimposed training and channel estimation for two-way relay networks (IEEE Pyongyang, South Korea, 2014), pp. 1050-1054

24. M Biguesh, AB Gershman, Training-based MIMO channel estimation: a study of estimator tradeoffs and optimal training signals. IEEE Trans. Signal Process. 54(3), 884-893 (2006)

25. TF Wong, B Park, Training sequence optimization in MIMO systems with colored interference. IEEE Trans. Commun. 52(11), 1939-1947 (2004)

doi:10.1186/1687-6180-2014-177

Cite this article as: El Astal and Olivier: Improved signal detection for twoway wireless relaying networks under imperfect time synchronisation. EURASIP Journal on Advances in Signal Processing 2014 2014:177.

\section{Submit your manuscript to a SpringerOpen ${ }^{\circ}$ journal and benefit from:}

- Convenient online submission

- Rigorous peer review

- Immediate publication on acceptance

- Open access: articles freely available online

- High visibility within the field

- Retaining the copyright to your article

Submit your next manuscript at $\boldsymbol{\wedge}$ springeropen.com 